\title{
Design of APOD-based switching dynamic observers and output feedback control for a class of nonlinear distributed parameter systems
}

\author{
Davood Babaei Pourkargar and Antonios Armaou ${ }^{1}$ \\ Department of Chemical Engineering, The Pennsylvania State University, University Park, PA 16802
}

\begin{abstract}
The output feedback control problem for a class of nonlinear distributed parameter systems with limited number of continuous measurement sensors that describes a wide range of physico-chemical systems is investigated using adaptive proper orthogonal decomposition (APOD) method. Specifically, APOD is used to initiate and recursively revise locally valid reduced order models (ROMs) that approximate the dominant dynamic behavior of such physico-chemical systems. The controller is designed based on ROMs by combining a robust state controller with an APOD-based nonlinear Luenberger-type switching dynamic observer of the system states to reduce measurement sensors requirements. The important static observer requirements on the number of measurement sensors (that must be supernumerary to the ROM dimension) and their location is circumvented by synthesizing dynamic observers. Three different approaches are introduced to recursively compute the dynamic observer gains at the ROM revisions. The stability of the closed-loop system is proven via Lyapunov and hybrid system stability arguments without invoking the separation principle between control and observation. The proposed method is successfully used to regulate a physico-chemical system that can be described in the form of the Kuramoto-Sivashinsky equation when the process exhibits significant nonlinear behavior.
\end{abstract}

Keywords: distributed parameter systems, output feedback control, adaptive model reduction, nonlinear systems, dynamic observer, process control

\section{Introduction}

Important processes in chemical and advanced material industry often couple complex transport phenomena with chemical reactions, such as reactive distillation in petrochemical industry, lithographic processes in semiconductor manufacturing, chemical vapor deposition processes, plasma discharge reactors, and tin float bath process in glass production $[1,18,39,61]$. In recent years, research in the field of process control has focused on the synthesis of control structures for these transport-reaction processes. The spatially distributed and nonlinear nature of the associated system dynamics make the control problem nontrivial $[1,15,18,38,44,52,53,58]$. The significant diffusion transport of the species leads to a mathematical description of dissipative partial differential equations (PDEs) whose long term and dominant behavior can be represented by finite number of the process spatial profiles [56] because the characteristic spectrum of the spatial differential operator of such PDEs can be decomposed to a finite-dimensional slow and an infinite-dimensional fast and stable parts [18].

\footnotetext{
${ }^{1}$ Corresponding author, Tel: +1(814)865-5316, Fax: +1(814)865-7846, Email address: armaou @engr.psu.edu
} 
Based on this property, the control problem of spatially distributed processes described by dissipative PDEs is usually addressed through model order reduction (MOR). MOR is based on deriving finitedimensional approximations of the infinite-dimensional representation of PDEs within appropriate functional spaces [18]. Modal decomposition techniques have been used to obtain ordinary differential equations (ODEs) which we term reduced order models (ROMs) [13, 14]. One method to obtain the ROM is using basis function expansion and the method of weighted residuals to derive infinite-dimensional representations that capture the system dynamics. The time-varying weights of the basis functions to express the system state are called modes of the state. Using singular perturbation arguments we obtain the finitedimensional ROM that presents the dominant system dynamics by considering a proper number of ODEs that correspond to the dominant basis functions of the system.

An issue that is commonly encountered is the number and type of the dominant basis functions that will be employed in order to obtain accurate lower order models. If not properly chosen, these approaches can lead to high dimensionality of the ROM and complicated controller designs. A common approach to identify the functional spaces and compute the set of basis functions is by solving the eigenfunction problem of the spatial differential operator for the PDE systems and using them as basis of the space. This problem cannot be solved analytically in general. As a result, common analytical model reduction approaches cannot be applied to the distributed parameter systems (DPSs) with nonlinear spatial differential operators or to processes defined over peculiar spatial domains such as corners and irregular geometries. Recent research efforts have focused on statistical techniques to address such issues. Statistical approaches use spatially distributed data obtained from the system to compute the set of dominant empirical basis functions $[45,61]$.

A well established approach to compute the set of basis functions is proper orthogonal decomposition (POD) [50]. POD is a large class of practical pattern recognition methods that obtain the dominant behavior of a system using an ensemble of data. POD-type methods have been widely applied in model order reduction, monitoring, control and optimization of DPSs [2, 3, 4, 5, 19, 29]. The essential requirement of the original POD algorithm and its variants to compute the optimal set of dominant empirical basis functions is a priori availability of large ensemble of solution data from the PDE system because the resulting POD-based empirical basis functions are only representative of the corresponding ensemble. Furthermore, the ensemble of solutions must be representative of the system solution globally and sufficiently excite all the modes of the system (persistence of excitation) and the ensemble average is equated with a time average over the solution (ergodicity). Then the usefulness of POD for certain systems is limited in practice. To relax the stringent conditions of POD methods, adaptive proper orthogonal decomposition (APOD) was developed to recursively revise the set of empirical basis functions as more information become available during process evolution [61]. Comparing to on-line POD methods, APOD leads to a three-fold increase in computational speed that makes this algorithm implementable [45]. Gappy-APOD was recently used for control of DPSs in the presence of partial distributed measurement sensors information [46]. A modified APOD methodology was also introduced for model reduction and control of dissipative nonlinear DPSs with fast transients [8]. APOD-based geometric observers and controllers was designed to track the output of nonlinear dissipative DPSs $[10,11]$. A criterion was identified to reduce the computational and measuring costs by minimizing the frequency of ROM revisions [9, 12].

While dynamic observer designs has reached a certain maturity for dynamic systems described by linear ODEs, this synthesis problem remains a difficult task for nonlinear ODE systems. The dynamic observer synthesis theory was developed by Luenberger for lumped systems described by a set of linear ODEs [41]. In last three decades, the problem of dynamic observer design for nonlinear systems 
has received considerable attention $[31,34,55]$. Several types of dynamic observers have been developed for nonlinear ODE systems' state estimation such as Luenberger-type [35], global exponential [33], Lyapunov-based [57], sampled-data nonlinear [32], high gain [28] and optimization-based [43] observers. Most of the dynamic observers introduced for PDE systems can be only applied on linear and bilinear PDEs [51, 63]. Efforts in design of linear dynamic observers for linear PDEs have focused on infinitedimensional Luenberger-type approaches in past years (see Ref. [22] and its references). Auxiliary functional observers are also used to stabilize PDEs using boundary observation [27]. Exponentially convergent observers are designed for a class of parabolic PDEs where only boundary sensors are available [51].

In this paper, we investigate the output feedback control problem of dissipative DPSs using limited number of continuous measurements sensors. Specifically, our effort focuses on the efficient design of switching dynamic observers based on adaptively updating ROMs and further on-line refinement from measurements during process evolution. ROM updating is also based on the efficient construction of basis functions. The important static observer requirements of available number of measurement sensors being supernumerary to the ROM dimension and required designs for the measurement sensors placement is circumvented using dynamic observer synthesis that conceptually needs only one measurement output to compute the estimation error and construct the dynamic observer structure. The combination of APOD with a dynamic observer design method that is stable in the Lyapunov sense allows us to synthesize nonlinear robust output feedback controllers that account for model reduction error and uncertainty, and guarantee local regulation of the process and output tracking. The assumption of separation principle is relaxed in controller/observer synthesis. During the controller/observer synthesis steps, first the design problem of output feedback controller and dynamic observer is investigated between ROM revisions and Lyapunov stability is proved in the time period between two model reconstructions. A supervisory scheme is subsequently designed to enforce closed-loop stability to ROM revisions procedure. The proposed control structure is illustrated on the stabilization problem of the Kuramoto-Sivashinsky equation (KSE) at an open-loop unstable steady state.

\section{Preliminaries}

\subsection{Class of nonlinear dissipative PDE}

We consider processes with a nonlinear dissipative, input-affine, PDE description in the following state space representation

$$
\begin{gathered}
\frac{\partial}{\partial t} \bar{x}(z, t)=\mathcal{A}(z) \bar{x}(z, t)+\mathcal{F}(z, \bar{x})+b(z) u(t), \\
y_{m}(t)=\int_{\Omega} s(z) \bar{x}(z, t) d z \\
y_{r}\left(z, t_{k}\right)=\int_{0}^{t} \delta\left(t-t_{k}\right) \bar{x}(z, t) d t
\end{gathered}
$$

subject to boundary conditions

$$
q\left(\bar{x}, \frac{\partial \bar{x}}{\partial z}, \ldots, \frac{\partial^{n_{0}-1} \bar{x}}{\partial z^{n_{0}-1}}\right)=0 \text { on } \partial \Omega
$$

and initial condition

$$
\bar{x}(z, 0)=\bar{x}_{0}(z)
$$


In the PDE system of (1)-(3), $t$ is the time, $z \in \Omega \subset \mathbb{R}^{3}$ denotes the spatial coordinate, $\Omega$ is the process domain with its boundary, $\partial \Omega$. The vector of state variables is denoted by $\bar{x}(z, t) \in \mathbb{R}^{n}$ and $u(t) \in \mathbb{R}^{l}$ is the vector of manipulated inputs. $\mathcal{A}(z)$ and $\mathcal{F}(z, \bar{x})$ present the dissipative linear and nonlinear parts of the system spatial differential operator of order $n_{0}$, respectively. Note that the nonlinear operator is bounded Lipschitz. $b(z) \in \mathbb{R}^{n \times l}$ is a smooth matrix function of $z$ that describes spatial distribution of the control actions. $q(\cdot)$ is a smooth vector function, $\left.\frac{\partial^{i} \bar{x}}{\partial z^{i}}\right|_{\partial \Omega}$ for $i=1, \ldots, n_{0}-1$, presents the spatial derivatives in the direction perpendicular to the process boundary of $\partial \Omega$ and $\bar{x}_{0}(z)$ is also a smooth vector function of $z$.

Two types of measurement outputs are assumed to be available for the process: (1) periodic distributed snapshot measurement output, $y_{r}\left(z, t_{k}\right) \in \mathbb{R}^{n}$, and (2) continuous measurement output, $y_{m} \in \mathbb{R}^{r}$, where $r$ indicates the number of continuous measurement sensors and $t_{k}$ shows the instance for snapshot measurements. The spatial distribution of the continuous measurement sensors is denoted by $s(z)$. Note that the results of the current work are presented for $\bar{x} \in \mathbb{R}$. We can extend the results for $\bar{x}=\left[\bar{x}_{1} \bar{x}_{2} \cdots \bar{x}_{n}\right]^{T} \in \mathbb{R}^{n}$, by considering each component of $\bar{x}_{i}$ for $i=1, \ldots, n$, individually [50]. The control objective is to stabilize the dissipative DPSs described by the PDE system of (1)-(3) at a desired spatial profile of $\bar{x}_{d}(z, t)$. To implement the proposed method in this paper we consider the spatially uniform steady state of $\bar{x}_{d}(z, t)=0$ as the desired profile.

\subsection{Infinite-dimensional representation}

To present the results, we formulated the PDE system of (1) as an infinite-dimensional system in an appropriate Hilbert subspace $\mathbb{H}(\Omega, \mathbb{R})$ where the space derivatives' definitions are added to the subspace. We also consider the inner product and norm in $L_{2}[\Omega]$ as

$$
\left(\vartheta_{1}, \vartheta_{2}\right)=\int_{\Omega} r(z) \vartheta_{1}^{T}(z) \vartheta_{2}(z) d z \quad \text { and } \quad\left\|\vartheta_{1}\right\|_{2}=\left(\vartheta_{1}, \vartheta_{1}\right)^{1 / 2}
$$

where $\vartheta^{T}$ is the transpose of $\vartheta$ and $r(z)$ denotes the spatially distributed weight function that is considered to be 1 in this work. By defining the state $x$ on $\mathbb{H}(\Omega, \mathbb{R})$ as

$$
x(t)=\bar{x}(z, t),
$$

the differential operators

$$
\begin{aligned}
\mathbf{A} x & =\mathcal{A}(z) \bar{x}, \\
\mathbf{F}(x) & =\mathcal{F}(z, \bar{x}),
\end{aligned}
$$

and the input and measured output operators as $\mathbf{B} u=b(z) u, S_{m} x=\int_{\Omega} s(z) \bar{x} d z$, the PDE system of (1) can be represented in the infinite-dimensional form

$$
\begin{gathered}
\dot{x}=\mathbf{A} x+\mathbf{F}(x)+\mathbf{B} u, \\
y_{m}=S_{m} x,
\end{gathered}
$$

with initial condition

$$
x(0)=x_{0} .
$$

Based on bounded locally Lipschitz assumption for $\mathcal{F}$, we can conclude that $\mathbf{F}$ is a nonlinear smooth vector function that satisfies $\mathbf{F}(0)=0$ and is also locally Lipschitz

$$
\left\|\mathbf{F}\left(x_{1}\right)-\mathbf{F}\left(x_{2}\right)\right\|_{2} \leq K_{L}\left\|x_{1}-x_{2}\right\|_{2}
$$


where $K_{L}$ is the Lipschitz gain in the above inequality.

Assumption 1 (state-space partitioning) The infinite-dimensional representation of the system (1)-(3) in an appropriate Hilbert subspace, $\mathbb{H}$, can be partitioned into a finite number of slow (possibly unstable) and an infinite number of fast and stable subsystems; also a time scale separation can be identified between the two subsystems dynamics.

The analytical basis functions needed to discretize the infinite-dimensional representation of the system in $\mathbb{H}$, can be computed from the eigenfunction problem of the operator $\mathbf{A}$,

$$
\mathbf{A} \phi_{i}=\lambda_{i} \phi_{i}, \quad i=1, \ldots, \infty
$$

subject to

$$
q\left(\phi_{i}, \frac{d \phi_{i}}{d z}, \ldots, \frac{d^{n_{0}-1} \phi_{i}}{d z^{n_{0}-1}}\right)=0 \text { on } \partial \Omega
$$

where $\lambda_{i}$ and $\phi_{i}$ denote the $i^{\text {th }}$ eigenvalue and the corresponding eigenfunction, respectively.

Assumption 1 restated The ordered eigenspectrum of the operator $\mathbf{A},\left\{\lambda_{1}, \lambda_{2}, \ldots\right\}$, can be decomposed to a finite-dimensional set of $p$ slow eigenvalues with possibly positive real parts, $\left\{\lambda_{1}, \lambda_{2}, \ldots, \lambda_{p}\right\}$, and an infinite-dimensional complement set of the remaining fast eigenvalues with negative real parts, $\left\{\lambda_{p+1}, \lambda_{p+2}, \ldots\right\}$ where the corresponding eigenfunctions to the two subsets of eigenvalues can be presented as $\Phi_{s}=\left[\begin{array}{llll}\phi_{1} & \phi_{2} & \cdots & \phi_{p}\end{array}\right]^{T}, \Phi_{f}=\left[\phi_{p+1} \phi_{p+2} \cdots\right]^{T}$. Also a time scale separation can be identified between slow and fast subsets of the eigenvalues as $\sigma=\frac{\left|\operatorname{Re}\left(\lambda_{1}\right)\right|}{\left|\operatorname{Re}\left(\lambda_{p+1}\right)\right|}$ where Re denotes the real part.

According to Assumption 1, we can decompose the Hilbert subspace, $\mathbb{H}$, to a slow subspace, $\mathbb{H}_{s} \triangleq$ $\operatorname{span}\left\{\phi_{i}\right\}_{i=1}^{p}$, and a complement fast and stable subspace, $\mathbb{H}_{f} \triangleq \operatorname{span}\left\{\phi_{i}\right\}_{i=p+1}^{\infty}$, i.e., $\mathbb{H}=\mathbb{H}_{s} \oplus \mathbb{H}_{f}$. Then by defining the orthogonal projection operators of $\mathcal{P}: \mathbb{H} \rightarrow \mathbb{H}_{s}, \mathcal{P}=\left(\cdot, \Phi_{s}\right)$ and $Q: \mathbb{H} \rightarrow \mathbb{H}_{f}, Q=\left(\cdot, \Phi_{f}\right)$, we obtain

$$
x=x_{s}+x_{f}
$$

where $x_{s}=\mathcal{P} x \in \mathbb{H}_{s}, x_{f}=Q x \in \mathbb{H}_{f}$.

Using such decomposition, the system of (6) can be discretized in the form of a partitioned ODE set of vectorized modes,

$$
\begin{gathered}
\dot{x}_{s}=A_{s} x_{s}+F_{s}\left(x_{s}, x_{f}\right)+B_{s} u, \\
\dot{x}_{f}=A_{f} x_{f}+F_{f}\left(x_{s}, x_{f}\right)+B_{f} u, \\
y_{m}=S_{m} x_{s}+S_{m} x_{f}, \\
y_{r}=S_{r} x_{s}+S_{r} x_{f},
\end{gathered}
$$

subject to the following initial conditions

$$
\begin{gathered}
x_{s}(0)=\mathcal{P} x(0)=\mathcal{P} x_{0}, \\
x_{f}(0)=Q x(0)=Q x_{0},
\end{gathered}
$$

where $A_{s}=\mathcal{P} \mathbf{A}\left(x_{s}+x_{f}\right)=\mathcal{P} \mathbf{A} x_{s}, A_{f}=Q \mathbf{A}\left(x_{s}+x_{f}\right)=Q \mathbf{A} x_{f}, F_{s}=\mathcal{P} \mathbf{F}, F_{f}=Q \mathbf{F}, B_{s}=\mathcal{P} \mathbf{B}, B_{f}=Q \mathbf{B}$.

Assumption 2 (Observability and controllability) The PDE system of (1)-(3) is assumed to be approximately observable and controllable [22]. 
Remark 1. The APOD algorithm requires the spatially distributed profile of the system states (snapshots) at revisions to revise the set of empirical basis functions during closed-loop process evolution. Consequently, we assume the existence of the periodic distributed snapshot measurement output, $y_{r}\left(z, t_{k}\right) \in \mathbb{R}^{n}$. Such required snapshots can be accessible from periodic fixed or moving spatially distributed measurement sensors, or be computed using in parallel high fidelity simulator. The required snapshots at each revisions in the presence of partial sensor information can be reconstructed by Gappy-APOD from previous spatially distributed profiles [46]. Gappy-APOD reconstructs the required data via a two tier procedure for a part of the spatial domain which was not measured. We can also address the snapshot construction problem by spatially distributed dynamic observers in the form of PDEs which only use a limited number of fixed point measurement sensors. The on-line snapshot construction problem to circumvent this basic assumption is the subject of the authors' current work.

Remark 2. The state-space partitioning assumption (Assumption 1) is satisfied by the majority of transportreaction processes (see the catalytic rod example at section 2, the catalytic packed-bed reactor example at section 4 in Ref. [21], chemical vapor deposition process [1, 40, 58] and plasma discharge reactor [39]). This assumption validates the existence of only a few dominant modes that describe the dynamics of the parabolic PDE system and enables us to approximate the PDE by a finite-dimensional ODE system.

Remark 3. The controllability and observability of DPSs depend on the locations of control actuators and measurement sensors, and specification of control objectives, while the controllability and observability of the ROMs of DPSs additionally depend on the discretization method used and the number and location of discretization nodes [48]. In general chemical engineering processes are over-designed in terms of observability where measurement sensors are placed in appropriate locations. One of the common approaches to actuator and sensor placement is to select the locations based on open-loop considerations to ensure controllability and observability. For further work on different aspects of actuator and sensor placement based on controllability/observability and optimal measurement methods see Refs. [6, 24, 59, 60] and the references therein.

\section{APOD-based model reduction and controller/observer synthesis}

We investigate the design problem of output feedback control and dynamic observer between ROM revisions; Lyapunov stability is proved in the time period between two consecutive snapshot arrivals and model reconstructions. Contrary to most of the data-driven model reduction approaches which use only open-loop system data, in the proposed APOD-based model reduction approach the required data are obtained during closed-loop system evolution. Thus, the resulting ROM accounts for the effects of controller actions on the process and the possible destabilizing effect they may have even if they were initially neglected. The dynamic observer and controller that are designed in this section will be refined based on stability of hybrid systems adding a supervisory control scheme.

\subsection{A brief review to APOD algorithm}

In this section we briefly explain how APOD is used to construct the empirical basis functions and update them on demand. Such empirical basis functions are essential to develop the ROMs of (18). 


\subsubsection{Off-line steps}

Let $v=\left\{\bar{x}\left(z, t_{k}\right)\right\}_{k=1}^{K}$ be the set of $K$ snapshots called the ensemble, where $\bar{x}\left(z, t_{k}\right)$ is the available snapshot at time $t_{k}$, in which case $\bar{x}\left(z, t_{k}\right)=y_{r}\left(z, t_{k}\right)$. The initial ensemble can be obtained from openloop process data while the controller is inactivate. We may also use off-line high-fidelity simulations of the governing PDEs. The off-line APOD is applied to initialize the set of empirical basis functions that capture the dominant ensemble patterns and to initiate an indicator that shows the relative contribution of such empirical basis functions to the entire energy of patterns inside the ensemble.

The covariance matrix of the ensemble, $C_{K}:=\left(v^{T}, v^{T}\right)$, is computed. The computationally expensive eigenfunction problem of the spatial differential operator in (9) is thus replaced by the eigenvector problem for the covariance matrix. Without loss of generality it is assumed that $m$ eigenmodes capture $\varepsilon$ of patterns energy of the ensemble, i.e. $\sum_{i=1}^{m} \lambda_{i} / \sum_{i=1}^{K} \lambda_{i} \geq \varepsilon$, where $\lambda_{1}>\lambda_{2}>\cdots>\lambda_{K}$. An orthonormal basis obtains as

$$
\begin{gathered}
Z=\left[\omega_{1} \omega_{2} \cdots \omega_{m}\right], Z \in \mathbb{R}^{K \times m} \\
\Lambda=\left[\lambda_{i}\right]_{i i}, i=1, \ldots, m
\end{gathered}
$$

where $\omega_{i}$ denotes the $i^{t h}$ eigenvector of $C_{K}$ that corresponds to the $i^{t h}$ eigenvalue, $\lambda_{i}$. Then the vector of basis functions, $\Psi=\left[\psi_{1} \psi_{2} \cdots \psi_{m}\right]^{T}$, is computed by a linear combination of the system snapshots,

$$
\Psi=\Lambda^{-1 / 2} Z^{T} v
$$

The resulting eigenspace of $C_{K}$ used to partition $\mathbb{H}$ into a dominant subspace, $\mathbb{P}$, that contains the empirical basis functions which capture $\varepsilon$ of pattern energy in the ensemble to approximate $\mathbb{H}_{s}$ and the orthogonal complement subspace, $\mathbb{Q}$, that contains the rest of the empirical basis functions to approximate the dominant part of $\mathbb{H}_{f}$ [56]. The orthogonal projection operators $P$ and $Q$ onto subspaces $\mathbb{P}$ and $\mathbb{Q}$ are obtained as

$$
P=Z Z^{T}, Q=I_{K}-Z Z^{T}
$$

onto subspaces $\mathbb{P}$ and $\mathbb{Q}$, respectively, where $I_{K}$ is the identity matrix. A detailed analysis of the off-line APOD has been presented in Refs. [45, 50, 61].

\subsubsection{On-line steps}

During system evolution, the recursive algorithm updates the set of empirical basis functions once new measurements become available from the process. Such orthonormal basis of subspace $\mathbb{P}$ is recursively revised, while the orthonormal basis of $\mathbb{Q}$ is computed from the fact that $\mathbb{Q}$ is the orthonormal complement of $\mathbb{P}$. The mentioned recursive process is computationally efficient as long as the dimension of $\mathbb{P}$ is small (which partly depends on choosing an appropriate value for $\varepsilon$ and the system characteristics).

Specifically, when a new snapshot is obtained from the closed-loop process, it is added to the ensemble and the oldest snapshot is removed, i.e., $k=1+k \bmod K$ where $\bmod$ denotes the modulus operator. Thus, the ensemble only includes the most recent snapshots in each time step. Then the covariance matrix of $C_{K}$ is revised based on the updated ensemble. Due to the covariance matrix updates, the set of eigenvalues is revised, the dominant eigenspace and the set of empirical basis functions are changed when needed. A flow chart illustrating the APOD approach and the associated algebraic computations are presented in Fig. 1 , where $\xi$ denotes the contribution of the dominant eigenvalue of $c_{q}=Q C_{K} Q$, namely $\lambda_{m+1}$, towards the total pattern energy of the ensemble, i.e. $\xi=\lambda_{m+1} / \sum_{i=1}^{m+1} \lambda_{i}$, and $H=Z^{T} C_{K} Z$ is an $m \times m$ matrix (see Refs. [61, 45] for additional details). 


\subsection{Finite-dimensional approximation using method of weighted residuals}

The finite-dimensional approximation of the infinite-dimensional PDE system of (1)-(3) can be derived by the method of weighted residuals from the empirical basis functions. $\bar{x}(z, t)$ can be generally described by an infinite weighted sum of a complete vectorized set of empirical basis functions $\Psi(z)$. The following approximation can be obtained by truncating the series expansion of $\bar{x}(z, t)$ up to order $m$ :

$$
\bar{x}(z, t) \simeq \sum_{k=1}^{m} \psi_{k}(z) a_{k}(t) \stackrel{m \rightarrow \infty}{\longrightarrow} \bar{x}(z, t)=\sum_{k=1}^{\infty} \psi_{k}(z) a_{k}(t)
$$

where $a_{k}(t)$ are known as system time-varying empirical modes. The following $m^{t h}$ order ODEs are derived by substituting (17) in the PDE system of (1)-(3), multiplying the PDE with the weighting functions, $\varphi(z)$, and integrating over $\Omega$ :

$$
\begin{gathered}
\sum_{k=1}^{m}\left(\varphi_{v}(z), \psi_{k}(z)\right) \dot{a}_{k}(t)=\sum_{k=1}^{m}\left(\varphi_{v}(z), \mathcal{A}(z) \psi_{k}(z)\right) a_{k}(t)+\left(\varphi_{v}(z), \mathcal{F}\left(z, \sum_{k=1}^{m} \psi_{k}(z) a_{k}(t)\right)\right)+\left(\varphi_{v}(z), b(z)\right) u \\
v=1, \ldots, m, \\
y_{m}=\sum_{k=1}^{m}\left(s(z), \psi_{k}(z)\right) a_{k}(t) .
\end{gathered}
$$

The method of weighted residuals reduces to Galerkin's projection when the weighting functions, $\varphi(z)$, are set to be the same as the empirical basis functions, $\psi(z)$. Then (18) can be presented in the compact form of

$$
\begin{gathered}
\dot{a}=A a+f(a)+B u, \\
y_{m}=C a .
\end{gathered}
$$

In the above equation, $A, B$ and $C$ denote constant matrices between revisions and $f$ is a Lipschitz nonlinear vector function, i.e.,

$$
\left\|f\left(a_{1}\right)-f\left(a_{2}\right)\right\| \leq K_{l}\left\|a_{1}-a_{2}\right\|,
$$

where $K_{l}$ indicates the upper bound gain in the Lipschitz inequality. In general, we must employ an overestimator for the range of values that we are interested in, in order to obtain a local bound. We also assume that the reduced order model of (19) keeps the observability and controllability properties of the original PDE system of (1)-(3) due to the set of globally recursively updated empirical basis functions.

Remark 4. Note that since the basis functions, $\Psi$, are periodically revised, the approximate systems we obtain are switching systems i.e., the form and dimensionality of the system changes during process evolution. Furthermore, the designed observer and controller will also be revised as well at each switching, resulting in a switched closed-loop system, even though the process is continuous.

\subsection{APOD-based dynamic observer design}

For the APOD-based ROM revision and dynamic observer design, we assume the periodic availability of the spatially distributed snapshots and continuous availability of measurements of the process from a limited number of point sensors. A static observer was designed to estimate the modes in the ROM of (18) required by the output feedback control structure $[45,61]$, however in such design the number of required point sensors must be supernumerary to the number of modes, otherwise the observer gives erroneous 
estimates. In this paper, we employ a exponentially stable Luenberger-type nonlinear observer design to circumvent this essential issue. Note that the Luenberger-type dynamic observers need only one point measurement sensor to formulate the estimation error and predict the dynamic behavior of the system modes.

We can define the spatially distributed estimation error by neglecting the fast and stable dynamics of the system as follows

$$
E(z, t)=\bar{x}_{e}(z, t)-\bar{x}_{s}(z, t),
$$

where $\bar{x}_{s}$ denotes the state of the slow subsystem and $\bar{x}_{e}$ is its estimated value. The slow part of the original system state can be obtained using time-space separation as

$$
\bar{x}_{s}(z, t)=\Phi_{s}^{T}(z) x_{s}(t)
$$

and the estimated state

$$
\bar{x}_{e}(z, t)=\Psi^{T}(z) \hat{a}(t),
$$

where $\Psi=\left[\begin{array}{llll}\psi_{1} & \psi_{2} & \ldots & \psi_{m}\end{array}\right]^{T}, \Phi_{s}=\left[\begin{array}{llll}\phi_{1} & \phi_{2} & \ldots & \phi_{p}\end{array}\right]^{T} ; m$ is the number of APOD-based empirical basis functions and $p$ denotes the number of analytical basis functions of the slow subsystem operator and $\hat{a}$ is the vector of estimated modes of (19). Note that the set of analytical basis functions of the system are unavailable and are used only for the system analysis and to expose the stability conditions needed. We can not define the observation error as the subtraction of $\hat{a}-x_{s}$, because the vectors of $x_{s}$ and $\hat{a}$ in Eqs. (22) and (23) may have different dimensions due to the possible difference between the numbers of analytical basis functions, $\Phi_{s}$, and empirical basis functions, $\Psi$.

Assumption 3 We assume that $\mathbb{H}_{s} \subseteq \mathbb{P}$, locally, where $\mathbb{H}_{s} \triangleq \operatorname{span}\left\{\phi_{i}\right\}_{i=1}^{p}$ denotes the local subspace of the slow and possibly unstable modes and $\mathbb{P} \triangleq \operatorname{span}\left\{\psi_{i}\right\}_{i=1}^{m}$ is empirical dominant subspace.

Note that Assumption 3 can be justified from the excitation of the higher order dynamics during the system progression. Based on this assumption, subspace $\mathbb{P}$ includes the slow Hilbert subspace $\mathbb{H}_{S}$ and may include a part of the fast Hilbert subspace $\mathbb{H}_{f}$. Then by defining a bounded map in the form of a linear transformation, we can connect the slow Hilbert subspace and subspace $\mathbb{P}$ and find the corresponding states. Such linear transformation changes at ROM revisions to conserve the properties of the subspaces during closed-loop process evolution. The bounded map is defined as follows

$$
\bar{x}_{s}=\Phi_{s}^{T} x_{s}=\Psi^{T} \mathcal{M} x_{s}=\Psi^{T} \tilde{a}
$$

where $\tilde{a}=\mathcal{M} x_{s}, \mathcal{M}: \mathbb{H}_{s} \mapsto \mathbb{P}$ and

$$
\mathcal{M}=\left(\Psi^{T}, \Phi_{S}^{T}\right)
$$

Note that based on Assumption 3, $\mathcal{M}=\left(\Psi^{T}, \Phi_{s}^{T}\right), \mathcal{M}: \mathbb{H}_{s} \mapsto \mathbb{P}$, is an injective linear transformation, i.e., if $\tilde{a}_{1}=\tilde{a}_{2}, \forall \tilde{a}_{1}, \tilde{a}_{2} \in \mathbb{P}$ then $x_{s, 1}=x_{s, 2}, \forall x_{s, 1}, x_{s, 2} \in \mathbb{H}_{s}$. The reverse transformation can also be defined as $\mathcal{M}^{\perp}=\left(\Phi_{s}^{T}, \Psi^{T}\right), \mathcal{M}^{\perp}: \mathbb{P} \mapsto \mathbb{H}_{s}$ such that $I=\mathcal{M}^{\perp} \mathcal{M}: \mathbb{H}_{S} \mapsto \mathbb{H}_{s}$ is a bijective map. An interesting fact about this transformation is that $\mathcal{M}^{\perp}=\mathcal{M}^{T}$.

Using the transformation, the slow dynamics of (12) can be represented within subspace $\mathbb{P}$ as

$$
\begin{gathered}
\dot{\tilde{a}}=A \tilde{a}+f(\tilde{a})+B u, \\
y_{m}=C \tilde{a},
\end{gathered}
$$


where $A=\mathcal{M} A_{s} \mathcal{M}^{\perp}, B=\mathcal{M} B_{s}$ and $C=S_{m} \mathcal{M}^{\perp}$ are constant matrices between ROM revisions and $f=$ $\mathcal{M} F_{S}$ is a Lipschitz nonlinear function. From the transformation and its reverse it is straightforward to show that $A, B$ and $C$ matrices, and $f$ nonlinear function in (26) are the same as $A, B$ and $C$ matrices, and $f$ nonlinear function used in (19).

Based on (26), the Luenberger-type dynamic observer is formulated as

$$
\dot{\hat{a}}=A \hat{a}+f(\hat{a})+B u+L\left(C \hat{a}-y_{m}\right)
$$

where $\hat{a}$ is the vector of estimated modes and $L$ denotes the observer gain. Then based on Assumption 1, the observation error can be defined as

$$
E=\bar{x}_{e}-\bar{x}_{s} \simeq \Psi^{T} e
$$

where $e=\hat{a}-\tilde{a}$ is the observer error with respect to empirical eigenmodes, and the observer error dynamics can be derived from (27) and (26)

$$
\dot{e}=(A+L C) e+[f(\hat{a})-f(\hat{a}-e)]
$$

The following Lyapunov function is considered

$$
V=V_{o}+V_{c}=\frac{\zeta_{o}}{2} e^{T} P_{o} e+\frac{\zeta_{c}}{2} \hat{a}^{T} P_{c} \hat{a}
$$

where $\tilde{V}_{o}$ is the observer Lyapunov function (OLF) and $V_{c}$ denotes the control Lyapunov function (CLF), $P_{o}$ and $P_{c}$ are positive definite matrices and $P_{o}$ has bounded Frobenius norm, $\left\|P_{o}\right\| \leq K_{p} ; \zeta_{o}$ and $\zeta_{c}$ are positive numbers. Then we can obtain the time derivative of the mentioned Lyapunov function as $\dot{V}=\dot{V}_{o}+\dot{V}_{c}$.

By setting the control objective at dynamic regulation of the observer, $\hat{a} \rightarrow 0$, and considering the

following CLF $V_{c}=\frac{\zeta_{c}}{2} \hat{a}^{T} P_{c} \hat{a}$, we assume a controller can be designed that forces the time derivative of the $\mathrm{CLF}, \dot{V}_{c}$ to be negative. Thus we need only consider the OLF to be negative, $\dot{V}_{o}<0$, at this point. We will revisit this assumption during the controller design step.

The question thus becomes how to find the observer gain, $L$, that stabilizes the system of (27) in the Lyapunov sense. Three different approaches are proposed as follows to address observer gain computation.

\subsubsection{Pole placement}

As mentioned previously we need only establish that $\dot{V}_{o}<0$. For $P_{o}=I$ we obtain

$$
\dot{V}_{o}=\zeta_{o} e^{T} \dot{e}=\zeta_{o}\left(e^{T}(A+L C) e+e^{T}[f(\hat{a})-f(\hat{a}-e)]\right)<0 .
$$

From the Lipschitz condition (20) we conclude

$$
e^{T}[f(\hat{a})-f(\hat{a}-e)] \leq\left\|e^{T}\right\|\|f(\hat{a})-f(\hat{a}-e)\| \leq\left\|e^{T}\right\| K_{l}\|e\|=K_{l}\|e\|^{2}=K_{l} e^{T} e .
$$

then using (32), the inequality of (31) can be stated as follows

$$
e^{T}(A+L C) e+e^{T}\left(K_{l} I\right) e<0 \Rightarrow e^{T}\left(A+L C+K_{l} I\right) e<0 \Rightarrow A+L C+K_{l} I<0 .
$$


By considering $A_{o}=A+K_{l} I$, we can solve the inequality problem to find the observer gain, $L$, using any pole placement technique. The closed-loop poles of the dynamic observer error (the eigenvalues of $\left.A_{c}=A_{o}+L C\right)$, can be placed at arbitrarily locations by solving the following equation

$$
\left|s I-\left(A_{o}+L C\right)\right|=p_{\text {des }}(s)
$$

for the observer gain matrix where $I$ denotes the identity matrix and $p_{d e s}$ is the characteristic polynomial of the desired observation poles.

\subsubsection{Linear optimal observer}

Another approach to compute the observer gain is via linear quadratic regulation (LQR). In this approach, Eq. (34) can be restated as

$$
\left|s I-\left(A_{o}+L C\right)\right|=\left|\left(s I-\left(A_{o}+L C\right)\right)^{T}\right|=\left|s I-\left(A_{o}^{T}+C^{T} L^{T}\right)\right|
$$

where $A_{o}^{T}$ and $C^{T}$ are the dual system matrices in (36) and $L^{T}$ denotes the gain matrix for the state feedback control problem of the dual system

$$
\begin{gathered}
\dot{\tilde{e}}=A_{o}^{T} \tilde{e}+C^{T} \tilde{u}, \\
\tilde{u}=L^{T} \tilde{e} .
\end{gathered}
$$

We design a linear quadratic feedback regulator [62] to solve the dual control problem and compute the observer gain. A state feedback regulator law can be synthesized via an optimization problem that minimizes the following quadratic objective function

$$
J(\tilde{u})=\int_{0}^{\infty}\left(\tilde{a}^{T} Q \tilde{a}+\tilde{u}^{T} R \tilde{u}+2 \tilde{a}^{T} N \tilde{u}\right) d t
$$

subject to the dual system dynamics of (36) and that $Q, R$ and $N$ are chosen to be positive definite matrices. The optimal gain matrix of $L^{T}$ then is computed as

$$
L^{T}=-\left(R^{-1}\left(B^{T} S+N^{T}\right)\right)^{T} \Rightarrow L=-\left(N+S^{T} B\right) R^{-T}
$$

where $S$ is the solution of the associated algebraic Riccati equation

$$
A_{o} S+S A_{o}^{T}-\left(S C^{T}+N\right) R^{-1}\left(C S+N^{T}\right)+Q=0 .
$$

\subsubsection{Linear matrix inequality (LMI)}

In this approach, the time derivative of the OLF of (30) is chosen to be

$$
\dot{\tilde{V}}_{o}=\frac{\zeta_{o}}{2}\left(\dot{e}^{T} P_{o} e+e^{T} P_{o} \dot{e}\right) .
$$

Using (29), the time derivative of $\tilde{V}_{o}$ (which we derive to be negative) is

$$
\begin{aligned}
\dot{\hat{V}}_{o} & =\frac{\zeta_{o}}{2}\left[\left(e^{T}\left(A^{T}+C^{T} L^{T}\right)+[f(\hat{a})-f(\hat{a}-e)]^{T}\right) P_{o} e+e^{T} P_{o}((A+L C) e+[f(\hat{a})-f(\hat{a}-e)])\right] \\
& =\frac{\zeta_{o}}{2}\left[e^{T}\left((A+L C)^{T} P_{o}+P_{o}(A+L C)\right) e+e^{T} P_{o}[f(\hat{a})-f(\hat{a}-e)]+\left(e^{T} P_{o}[f(\hat{a})-f(\hat{a}-e)]\right)^{T}\right]<0 .
\end{aligned}
$$


Based on the Lipschitz condition (20) and bounded norm of $P_{o}$ we obtain

$$
\begin{aligned}
e^{T} P_{o}[f(\hat{a})-f(\hat{a}-e)]+\left(e^{T} P_{o}[f(\hat{a})-f(\hat{a}-e)]\right)^{T} & \leq 2\left\|e^{T} P_{o}[f(\hat{a})-f(\hat{a}-e)]\right\| \\
& \leq 2\left\|e^{T}\right\|\left\|P_{o}\right\|\|[f(\hat{a})-f(\hat{a}-e)]\| \\
& \leq 2 K_{l} K_{p}\left\|e^{T}\right\|\|e\| \\
& \leq K_{0} e^{T} e,
\end{aligned}
$$

where $K_{0}=2 K_{l} K_{p}$. Then inequality (41) can be expressed as follows using (42)

$$
e^{T}\left((A+L C)^{T} P_{o}+P_{o}(A+L C)\right) e+K_{0} e^{T} e<0 \Rightarrow e^{T}\left((A+L C)^{T} P_{o}+P_{o}(A+L C)+K_{0} I\right) e<0 .
$$

Since $P_{o} A_{c}+A_{c}^{T} P_{o}+K_{0} I<0$ where $A_{c}=A+L C$, it follows that $\dot{\tilde{V}}<0$ for all $e \neq 0$ and stability is proved. So $P_{o}$ and $L$ are chosen so that the following LMI is satisfied

$$
A_{c}^{T} P_{o}+P_{o} A_{c}+K_{0} I<-P_{o} W P_{o}-P_{o} L U L^{T} P_{o}
$$

where $W$ and $U$ are the design weighting matrices and they must symmetric positive definite.

From (44) we have

$$
\begin{aligned}
& A_{c}^{T} P_{o}+P_{o} A_{c}+P_{o} W P_{o}+P_{o} L U L^{T} P_{o}+K_{0} I<0 \\
& \Rightarrow\left(A^{T}+C^{T} L^{T}\right) P_{o}+P_{o}(A+L C)+P_{o} W P_{o}+P_{o} L U L^{T} P_{o}+K_{0} I<0 \\
& \Rightarrow A^{T} P_{o}+P_{o} A+\left(P_{o} L C\right)^{T}+P_{o} L C+P_{o} W P_{o}+P_{o} L U L^{T} P_{o}+K_{0} I<0 .
\end{aligned}
$$

Using $Y=P_{o} L$, (45) can be expressed as

$$
\begin{aligned}
& A^{T} P_{o}+P_{o} A+(Y C)^{T}+Y C+P_{o} W P_{o}+Y U Y^{T}+K_{0} I<0 \\
\Rightarrow & P_{o} A+A^{T} P_{o}+\left(Y^{T}+U^{-1} C\right)^{T} U\left(Y^{T}+U^{-1} C\right)-C^{T} U^{-1} C+P_{o} W P_{o}+K_{0} I<0
\end{aligned}
$$

By setting $Y^{T}=-U^{-1} C$ we have

$$
P_{o} A+A^{T} P_{o}-C^{T} U^{-1} C+P_{o} W P_{o}+K_{0} I<0 .
$$

The above matrix inequality is equivalent to

$$
\left(\begin{array}{cc}
P_{o} A+A^{T} P_{o}-C^{T} U^{-1} C+K_{0} I & P_{o} \\
P_{o} & -W^{-1}
\end{array}\right)<0
$$

using the Schur Complement Lemma [49].

The observer gain is computed by minimizing trace $\left(P_{o}^{-1}\right)$ subject to (48). The observer gain then can be directly computed as

$$
L=-P_{o}^{-1} C^{T} U^{-1}
$$


Proposition 1 The observation error using dynamic observer of (27) is locally stable while f satisfies the Lipschitz condition (20) and

(i) The characteristic polynomial roots are negative and feedback gain L is computed from (34) for the case when $P_{o}=I$,

(ii) The Riccatti equation of (39) has a solution, $S$, for positive definite matrices, $Q, R, N$ and feedback gain L is obtained from (38),

(iii) $W$ and $U$ are symmetric positive definite matrices and $L$ is obtained from (49).

Proof Let $V_{o}(e)=\frac{\zeta_{o}}{2} e^{T} P_{o}$ e be the OLF for observer error dynamics of (29) with the equilibrium point of $e=0$, where the dynamic observer of system described by $\left(27 . V_{o}(0)=0\right.$ and $V_{o}(e)>0$ in $\mathbb{R}^{m}-\{0\}$ where $m$ denotes the number of the modes between ROM revisions. Considering the observer gain matrix, L, computed by either

(i) Eq. (34), where the characteristic polynomial roots are negative and $P_{o}=I$,

(ii) Eq. (38), where the Riccatti equation of (39) has a solution, $S$ for positive definite matrices, $Q, R, N$,

(iii) Eq. (49), where $W$ and $U$ are symmetric positive definite matrices,

and assuming that $f$ satisfies the Lipschitz condition (20), we conclude that $\dot{V}_{o}(e)<0$ and $\dot{V}_{o}(0)=0$.

Based on Lyapunov's stability theorem (Theorem 4.1 in Ref. [37]), $\exists \delta_{o}>0:\|e(0)\|<\delta_{o}$, then the observer error dynamics of (29) are locally asymptotically stable.

Theorem 1 Consider the nonlinear dissipative, input-affine, PDE system of (1)-(3) with infinite-dimensional representation of (6) and finite-dimensional approximation of (26) in subspace $\mathbb{P}$ with APOD-based empirical basis functions when the time interval between ROM updates, $\delta$, is finite and larger than a critical value, $t_{b}$. Under Assumptions of 1-3 and Lipschitz assumption of nonlinear functions, the observation error dynamics is locally asymptotically stable between ROM revisions using dynamic observer of (27), with observer gain computed by either

(i) Eq. (34) where $P_{o}=I$ and Hurwitz characteristic polynomial,

(ii) Eq. (38) for positive definite matrices of $Q, R$ and $N$,

(iii) Eq. (49) for symmetric positive definite matrices of $W$ and $U$.

Proof The slow system observer error with respect to modes in subspace $\mathbb{H}_{s}$ can be defined as follows

$$
\hat{e}=\hat{x}_{s}-x_{s}
$$

where $\hat{e}=\mathcal{M}^{\perp}$ e and $\hat{x}_{s}=\mathcal{M}^{\perp} \hat{a}$.

From (29) and (50) we obtain

$$
\dot{\hat{e}}=\left(A_{s}+\mathcal{M}^{\perp} L S_{m}\right) \hat{e}+\left(F_{s}\left(\hat{x}_{s}, x_{f}\right)-F_{s}\left(\hat{x}_{s}-\hat{e}, x_{f}\right)\right) .
$$

Under Assumption 1 (time scale separation part), the system of (12) can be presented in the following singular perturbation form (sections 4.4 and 4.5 in Ref. [18]) augmented with the observation error dynamics of (51)

$$
\begin{gathered}
\dot{x}_{s}=A_{s} x_{s}+F_{s}\left(x_{s}, x_{f}\right)+B_{s} u, \\
\sigma \dot{x}_{f}=\sigma A_{f} x_{f}+\sigma\left(F_{f}\left(x_{s}, x_{f}\right)-B_{f} u\right), \\
\dot{\hat{e}}=\left(A_{s}+\mathcal{M}^{\perp} L S_{m}\right) \hat{e}+\left(F_{s}\left(\hat{x}_{s}, x_{f}\right)-F_{s}\left(\hat{x}_{s}-\hat{e}, x_{f}\right)\right),
\end{gathered}
$$


where $\sigma=\left|\lambda_{1}\right| /\left|\lambda_{p+1}\right|$ is a small value that indicates the separation between the dominant and nondominant dynamic behavior. From the definition of $A_{f}$ and $\sigma, A_{f \sigma}=\sigma A_{f}$ has eigenvalues with negative real parts of $O(1)$. We assume that $F_{f}\left(x_{s}, x_{f}\right)+B_{f} u$ does not locally include terms of $O\left(\frac{1}{\sigma}\right)$ [18], where $O(\cdot)$ indicates the order of $(\cdot)$. By defining $\tau=t / \sigma$ and setting $\sigma=0$, we can express the fast dynamics of (52) in the fast time scale form

$$
\frac{\partial x_{f}}{\partial \tau}=A_{f \sigma} x_{f}
$$

Thus, the solution can be obtained as

$$
x_{f}(\tau)=x_{f}(0) e^{A_{f \sigma} \tau} .
$$

Then from (54) and Schwartz inequality we conclude

$$
\left\|x_{f}(\tau)\right\|_{2}=\left\|x_{f}(0) e^{A_{f \sigma} \tau}\right\|_{2} \leq\left\|x_{f}(0)\right\|_{2}\left\|e^{A_{f \sigma} \tau}\right\|_{2} .
$$

Considering the eigenspectrum bound to the fast dynamics implies that

$$
\left\|x_{f}(0)\right\|_{2}\left\|e^{A_{f \sigma} \tau}\right\|_{2} \leq\left\|x_{f}(0)\right\|_{2} e^{\left(\sigma \lambda_{p+1}\right) \tau},
$$

where $\lambda_{p+1}$ denotes the dominant, least negative eigenvalue of $A_{f}$ which is of $O\left(\frac{1}{\sigma}\right)$. From (55), (56) and $\sigma=\left|\lambda_{1}\right| /\left|\lambda_{p+1}\right|$ we can obtain

$$
\left\|x_{f}(0)\right\|_{2} e^{\left(\sigma \lambda_{p+1}\right) \tau}=\left\|x_{f}(0)\right\|_{2} e^{-\left|\lambda_{1}\right| \tau} \leq O(\sigma)
$$

this bound is satisfied when

$$
\tau_{b}=\frac{t_{b}}{\sigma} \geq-\frac{1}{\left|\lambda_{1}\right|} \ln \left[O\left(\frac{\sigma}{\left\|x_{f}(0)\right\|_{2}}\right)\right] .
$$

Thus,

$$
t_{b} \geq \frac{1}{\left|\lambda_{p+1}\right|} \ln \left[O\left(\frac{\left\|x_{f}(0)\right\|_{2}}{\left|\lambda_{1}\right|}\left|\lambda_{p+1}\right|\right)\right] .
$$

We choose $\delta t$ such that $\delta t>t_{b}$ where $t_{b}$ denotes the time needed for for the fast dynamics of (52) to be stabilized and $\delta t=t_{k}-t_{k-1}$ is the time interval between ROM revisions. Then the observation error can be expressed as follows

$$
E_{o}=\hat{x}_{s}-x=\hat{x}_{s}-\left(x_{s}+x_{f}\right)=\hat{x}_{s}-\left(x_{s}+0\right)=\hat{x}_{s}-x_{s}=\hat{e},
$$

when the infinite-dimensional system of (52) is approximated by the finite-dimensional slow system for $t \geq t_{b}$

$$
\begin{gathered}
\dot{x}_{s}=A_{s} x_{s}+F_{s}\left(x_{s}, 0\right)+B_{s} u \\
\dot{\hat{e}}=\left(A_{s}+\mathcal{M}^{\perp} L S_{m}\right) \hat{e}+\left(F_{s}\left(\hat{x}_{s}, 0\right)-F_{s}\left(\hat{x}_{s}-\hat{e}, 0\right)\right) .
\end{gathered}
$$

Based on Proposition 1 we obtain that the OLF, $V_{o}$, is negative definite, thus observer error dynamics in (29) is locally asymptotically stable based on Lyapunov's stability theorem, i.e., for $\|e(0)\|<\delta_{o}$ there is a $\delta_{o}^{\prime}=\left\|\mathcal{M}^{\perp}\right\|_{2} \delta_{o}$ the dynamics of observer error of $E_{o}=\hat{e}$ is locally asymptotically stable in the Lyapunov sense such that $\left\|E_{o}\right\|_{2}=\|\hat{e}\|_{2}<\delta_{o}^{\prime}$.

The dynamic observer of (27) is redesigned when the ROM is updated by APOD. Thus, stability theorems of hybrid systems are needed to prove that the switching closed-loop system still remains stable 
during the periodic revisions of the ROM. Then multiple OLFs in the form of (30) must be considered for such hybrid system analysis [20]. Considering finite time intervals between ROM revisions, the negative time derivative of the multiple Lyapunov functions of (30) guarantees stability of the switching system (Theorem 3.2 in Ref. [23]) when the following condition [20, 23] is also satisfied

$$
V_{o}\left(e\left(t_{k}\right)\right)<V_{o}\left(e\left(t_{k-1}\right)\right)
$$

In the above condition, $k>1$ and $V_{o}\left(e\left(t_{k}\right)\right)$ corresponds to the value of Lyapunov function at the beginning of interval $\left[t_{k}, t_{k+1}\right]$. Note that the time interval between ROM revisions, $\delta t=t_{k+1}-t_{k}$, is chosen to be large enough to relax the fast dynamics of the system to zero.

The OLF, $V_{o}=\frac{\zeta_{o}}{2} e^{T} P_{o} e$, may possibly increase during dimensionality changes of the ROM. Thus, value $\zeta_{o}$ must be chosen appropriately from a supervisory logic in the closed-loop process.

We can directly compute the eigenmodes of the system at the revisions when the new snapshot arrives by the periodic snapshot measurements,

$$
\bar{x}\left(z, t_{k}\right)=\sum_{j=1}^{m} \psi_{j}(z) \tilde{a}_{j}\left(t_{k}\right) \Rightarrow \tilde{a}_{j}\left(t_{k}\right)=\int_{\Omega} \psi_{j}(z) \bar{x}\left(z, t_{k}\right) d z, \quad j=1, \ldots, m,
$$

where $x\left(z, t_{k}\right)=y_{r}\left(z, t_{k}\right)$ is the system snapshot at revision instant, $t_{k}$, and $m$ denotes the number of eigenmodes. Thus, the following equation is applied to update the value of $\zeta_{o}$

$$
\zeta_{o}=\xi \frac{e^{T}\left(t_{k-1}\right) e\left(t_{k-1}\right)}{e^{T}\left(t_{k}\right) e\left(t_{k}\right)},
$$

where $e=\hat{a}-\tilde{a}$. Alternatively, we initialize $\zeta_{o}$ at value $\zeta_{\text {in }}$ and reevaluate it using (63) only when the criteria of (62) is violated. This procedure leads to a less aggressive dynamic observer design that has smaller observer gain while guaranteeing stability of observation error dynamics.

Remark 5. The LMI approach is well defined but computationally expensive. The pole placement technique is fast but it can be ill-conditioned if unrealistic locations have been chosen for closed-loop poles. Generally we should avoid placing multiple poles at the same location and typically require high gain, L, when the open-loop poles are weakly observable which in turn sensitizes the entire closed-loop structure to perturbations [30].

Remark 6. For the ROM states to be unobservable the most probable situation is that all point sensors must be located at the zeros of a specific basis function with a corresponding decoupled eigenmode; this can in principle happen but very rarely, and as the number of sensors increases it becomes highly improbable. We can identify such cases on-line by comparing the positions of zeros of the computed basis functions and the placement of the sensors. Another reason for unstable estimates of the process state is the ROM/PDE model mismatch; this is the underlying reason we recursively update the ROM during process evolution.

\subsection{APOD-based output feedback controller design}

In this section, we now focus on synthesizing a controller structure that achieves the objective of regulating the system of (1) to the origin based on an appropriate finite-dimensional approximation of (26) 
using Lyapunov's direct method when the state values' estimates originate from dynamic observer (27). As described before, the ROM of (19) is an approximation of the main PDE system of (1). Due to this fact the model reduction error between ROM and original PDE is considered as system uncertainty that affects controller design. We should note that the assumption of separation principle between the controller and the observer is relaxed. A simpler controller design under separation principle is presented in Ref. [7].

In the proposed control structure, the ROM is used as the basis for output feedback controller synthesis, however, the state values needed by the controller to compute the control action are estimated from the dynamic observer. There is a difference (in the form of the Luenberger term) between the ROM equations used by the controller and the dynamic observer. If we assume principle of separation, there is no need to account for this term in the closed-loop stability analysis, because the principle of separation of observation and control implies the stability properties of the output feedback controller can be deduced from the stability analysis of the dynamic observer and deterministic state feedback controller, separately.

For synthesizing a control structure (a) without assuming separation principle between control and observation, and (b) to compensate for the uncertainty results from model reduction error, first we design the controller to stabilize the dynamic observer and then the stable dynamic observer to guarantee the observation error regulation. We consider the Luenberger term in (27) as a general non-vanishing perturbation. We also assume this perturbation can be considered as a summation of possibly time varying but bounded uncertain variables. By considering the internal uncertainty in the observer dynamics we can also circumvent the issues of ignoring the observation of fast and stable dynamics in (12) in the time period of $\left[0, t_{b}\right]$ that is discussed in the proof of Theorem 1 . In this case the system of (27) can be considered in the following general form

$$
\dot{\hat{a}}=F(\hat{a})+B u+\sum_{i=1}^{s} w_{i}(\hat{a}) \theta_{i}(t),
$$

where $F(\hat{a})=A \hat{a}+f(\hat{a}), L\left(C \hat{a}(t)-y_{m}(t)\right)=\sum_{i=1}^{s} w_{i}(\hat{a}) \theta_{i}(t), \theta_{i}(t) \in \Theta,\left\|\theta_{i}(t)\right\| \leq \theta_{b i}$ and $\Theta \subset \mathbb{R}$ is a nonempty compact convex subset. The upper bounds of $\theta_{b i}$ can be approximated based on monitoring the dynamic observer error and the norm of observer gain.

The vector of uncertain variables in (64) can compensate for uncertainty in the system due to basis functions computation or model mismatch and also can account for exogenous disturbances. Assuming that the perturbation is non-vanishing, the origin of the system is no longer an equilibrium point for the uncertain system. Thus, the objective is to synthesize a robust nonlinear output feedback controller that guarantees global boundedness in a neighborhood around the desired steady state.

Theorem 2 Consider the nonlinear dissipative PDE system in (1), for which Assumptions 1-3 hold. The following static output feedback control law [26, 54] asymptotically stabilizes the system of (1) when the time interval between ROM updates, $\delta$, is finite and larger than a critical value, $t_{b}$.

$$
u(t)=-k\left(\hat{a}, c_{o}, \rho, \chi, \theta_{b}\right)\left(L_{B} V_{c}\right),
$$

where $c_{o}>0, \rho>0$ and $\chi>1$ are adjustable parameters and

$$
k\left(\hat{a}, c_{o}, \rho, \chi, \theta_{b}\right)= \begin{cases}c_{o}+\frac{L_{F}^{\S} V_{c}+\sqrt{\left(L_{F}^{\S} V_{c}\right)^{2}+\left(\left\|\left(L_{B} V_{c}\right)\right\|\right)^{4}}}{\left(\left\|\left(L_{B} V_{c}\right)\right\|\right)^{2}}, & L_{B} V_{c} \neq 0 \\ c_{o}, & L_{B} V_{c}=0\end{cases}
$$




$$
L_{F}^{\S} V_{c}=L_{F} V_{c}+\rho\left\|P_{c} \hat{a}\right\|+\chi \sum_{i=1}^{s}\left\|L_{w_{i}} V_{c}\right\| \theta_{b i}
$$

$V_{c}=\frac{\zeta_{c}}{2} \hat{a}^{T} P_{c} \hat{a}$ is the CLF, $P_{c}$ is a positive definite matrix, $L_{B} V_{c}=\left[L_{B_{1}} V_{c} \ldots L_{B_{l}} V_{c}\right]^{T}$ is a column vector, $L_{F} V_{c}=\frac{\partial V_{c}}{\partial \hat{a}} F, L_{B} V_{c}=\frac{\partial V_{c}}{\partial \hat{a}} B$ denote Lie derivatives and $\theta_{b}=\left[\begin{array}{llll}\theta_{1} & \theta_{2} & \cdots & \theta_{s}\end{array}\right]^{T}$.

Proof Consider the nonlinear uncertain system of (64) whose CLF is defined as follows

$$
V_{c}=\frac{\zeta_{c}}{2} \hat{a}^{T} P_{c} \hat{a}
$$

where $P_{c}$ is a positive definite matrix. It is obvious that $V_{c}(0)=0$ and $V_{c}(\hat{a})>0$ in $\mathbb{R}^{m}-\{0\}$.

By substituting Eqs. (64) and (65) in the CLF time derivative, we obtain

$$
\begin{aligned}
\dot{V}_{c}=L_{F} V_{c}+L_{B} V_{c} u+\sum_{i=1}^{s} L_{w_{i}} V_{c} \theta_{i}= & -c_{o}\left(\left\|L_{B} V_{c}\right\|\right)^{2}-\rho\left\|P_{c} \hat{a}\right\|-\chi \sum_{i=1}^{s}\left\|L_{w_{i}} V_{c}\right\| \theta_{b i} \\
& -\sqrt{\left(L_{F} V_{c}+\rho\left\|P_{c} \hat{a}\right\|+\chi \sum_{i=1}^{s}\left\|L_{w_{i}} V_{c}\right\| \theta_{b i}\right)^{2}+\left(\left\|\left(L_{B} V_{c}\right)\right\|\right)^{4}} \\
& +\sum_{i=1}^{s} L_{w_{i}} V_{c} \theta_{i} .
\end{aligned}
$$

Using $\sum_{i=1}^{s} L_{w_{i}} V_{c} \theta_{i} \leq \sum_{i=1}^{s}\left\|L_{w_{i}} V_{c}\right\| \theta_{b i}$ and by adjusting the parameters as $\rho>0$ and $\chi>1$, the time derivative of the CLF is negative, $\dot{V}_{c}<0$. Thus $\exists \delta_{c}>0:\|\hat{a}(0)\|<\delta_{c}$, that the closed-loop system in (64) is locally asymptotically stable in the Lyapunov sense (Theorem 4.1 in Ref. [37]).

In section "APOD-based dynamic observer" we assumed that if a controller can be synthesized such that $\dot{V}_{c}<0$, based on the stability of the dynamic observer the closed-loop process will be asymptotically stable, i.e. $e$ and $\hat{a}$ will be bounded. From the definition $e=\hat{a}-\mathcal{M}$ a and the fact that we did not use the separation principle we conclude that a will be bounded.

Substituting the controller (65) into the Hilbert representation of the infinite-dimensional system (12) and dynamic observer of (27), we obtain

$$
\begin{gathered}
\dot{x}_{s}=A_{s} x_{s}+F_{s}\left(x_{s}, x_{f}\right)-B_{s} k\left(\hat{a}, c_{o}, \rho, \chi, \theta_{b}\right)\left(L_{B} V_{c}\right), \\
\dot{x}_{f}=A_{f} x_{f}+F_{f}\left(x_{s}, x_{f}\right)-B_{f} k\left(\hat{a}, c_{o}, \rho, \chi, \theta_{b}\right)\left(L_{B} V_{c}\right), \\
\dot{\hat{a}}=A \hat{a}+f(\hat{a})-B k\left(\hat{a}, c_{o}, \rho, \chi, \theta_{b}\right)\left(L_{B} V_{c}\right)+L\left(C \hat{a}-S_{m} x_{s}-S_{m} x_{f}\right) .
\end{gathered}
$$

Based on Assumption 1, (68) can be stated in the following singular perturbation form (sections 4.4 and 4.5 in Ref. [18])

$$
\begin{gathered}
\dot{x}_{s}=A_{s} x_{s}+F_{s}\left(x_{s}, x_{f}\right)-B_{s} k\left(\hat{a}, c_{o}, \rho, \chi, \theta_{b}\right)\left(L_{B} V_{c}\right), \\
\sigma \dot{x}_{f}=\sigma A_{f} x_{f}+\sigma\left(F_{f}\left(x_{s}, x_{f}\right)-B_{f} k\left(\hat{a}, c_{o}, \rho, \chi, \theta_{b}\right)\left(L_{B} V_{c}\right)\right), \\
\dot{\hat{a}}=A \hat{a}+f(\hat{a})-B k\left(\hat{a}, c_{o}, \rho, \chi, \theta_{b}\right)\left(L_{B} V_{c}\right)+L\left(C \hat{a}-S_{m} x_{s}-S_{m} x_{f}\right),
\end{gathered}
$$

where $\sigma=\frac{\left|\operatorname{Re}\left(\lambda_{1}\right)\right|}{\left|\operatorname{Re}\left(\lambda_{p+1}\right)\right|}$ is a small value that presents the separation between the dominant and non-dominant eigenmodes. $\lambda_{1}$ and $\lambda_{p+1}$ are the dominant eigenvalues of slow and stable fast subsets of eigenvalues of $\mathbf{A}$, 
respectively. Based on the control law (65) and assuming bounded control action and Lipschitz condition for $\mathbf{F},(8)$, then $F_{f}\left(x_{s}, x_{f}\right)-B_{f} k\left(\hat{a}, c_{o}, \rho, \chi, \theta_{b}\right)\left(L_{B} V_{c}\right)$ does not have a term of $O\left(\frac{1}{\sigma}\right)$ [18].

By defining $\tau=\frac{t}{\sigma}$, it directly results from the definitions that $A_{f \sigma}=\sigma A_{f}$ is of order $O(1)$. Then by setting $\sigma=0$, the fast subsystem of (69) can be presented in the following locally exponentially stable subsystem

$$
\frac{\partial x_{f}}{\partial \tau}=A_{f \sigma} x_{f}
$$

Based on singular perturbation theory [18] after a period of time, $t_{b}$, where

$$
t_{b} \geq \frac{1}{\left|\lambda_{p+1}\right|} \ln \left[O\left(\frac{\left\|x_{f}(0)\right\|_{2}}{\left|\lambda_{1}\right|}\left|\lambda_{p+1}\right|\right)\right],
$$

the fast dynamics of the system stabilize to zero, $x_{f} \simeq 0$ and the closed-loop PDE system of (69) is approximated by the finite-dimensional slow system below

$$
\begin{gathered}
\dot{x}_{s}=A_{s} x_{s}+F_{s}\left(x_{s}, 0\right)-B_{s} k\left(\hat{a}, c_{o}, \rho, \chi, \theta_{b}\right)\left(L_{B} V_{c}\right) . \\
\dot{\hat{a}}=A \hat{a}+f(\hat{a})-B k\left(\hat{a}, c_{o}, \rho, \chi, \theta_{b}\right)\left(L_{B} V_{c}\right)+L\left(C \hat{a}-S_{m} x_{s}\right) .
\end{gathered}
$$

The system of (72) is stabilized by the controller/observer design.

At the ROM revisions, the same strategy for dynamic observer designs is used for $\zeta_{c}$ because CLF, $V_{c}=\frac{\zeta_{c}}{2} \hat{a}^{T} P_{c} \hat{a}$, also may increase during the ROM dimensionality changes. The value of $\zeta_{c}$ must be chosen appropriately to satisfy the hybrid systems stability criteria [20]. A possible strategy is to automatically adjust $\zeta_{c}$ at every instance of ROM changes as follows

$$
\zeta_{c}=\xi \frac{\hat{a}^{T}\left(t_{k-1}\right) \hat{a}\left(t_{k-1}\right)}{\hat{a}^{T}\left(t_{k}\right) \hat{a}\left(t_{k}\right)}
$$

in order to retain an aggressive controller throughout the process operation. The strategy used in the current work is to initialize $\zeta_{c}$ at value $\zeta_{\text {in }}$ and reevaluate it using (73) only when constraints of (62) are violated. This procedure leads to less aggressive control action while guaranteeing closed-loop stability.

Theorem 3 The DPS of (1) that is described by the switching ROM of (26), can be asymptotically stabilized by the output feedback control structure of (65) and (27) using supervisory strategies of (73) and (63) to evaluate $\zeta_{c}$ and $\zeta_{o}$ at each time period when the time interval between the ROM revisions, $\delta$, is chosen to satisfy $\delta t>t_{b}$, where $t_{b}$ is obtained from (58).

Proof Based on Proposition 1 and Theorems 1, 2 we obtained

$$
\dot{V}_{o}<0, \quad \dot{V}_{c}<0,
$$

between ROM revisions and the observer/controller structure redesigns. As a result the closed-loop system will destabilize only when $A, B, C$ and $f$ in (26) and (19) deviate due to appearance of new trends. In such case the structure of the ROM is revised using APOD methodology. According to such system structure changes the extra Lyapunov stability condition of switching systems must be satisfied to guarantee the 
stability of the close-loop system (Theorem 3.2 in Ref. [23]).

The supervisory strategies of (73) and (63) directly enforce the Lyapunov stability criterion for of the switching systems, (62), in the controller and observer designs thus the OLF and CLF satisfy the conditions of (62). It means that during the ROM revisions the $\zeta_{c}$ and $\zeta_{o}$ values are periodically chosen subject to $V_{c}\left(\hat{a}\left(t_{k}\right)\right)$ and $V_{o}\left(e\left(t_{k}\right)\right)$.

Then $\dot{V}<0$ between $R O M$ revisions as follows

$$
\dot{V}=\dot{V}_{o}+\dot{V}_{c}<0
$$

Thus $\exists \delta>0:\|a(0)\|<\delta$, that the closed-loop system is locally asymptotically stable in the Lyapunov sense (Theorem 4.1 in Ref. [37]).

Also the closed-loop Lyapunov function satisfies the criteria (62),

$$
V\left(t_{k}\right)=V_{c}\left(\hat{a}\left(t_{k}\right)\right)+V_{o}\left(\hat{a}\left(t_{k}\right)\right)<V_{c}\left(\hat{a}\left(t_{k-1}\right)\right)+V_{o}\left(\hat{a}\left(t_{k-1}\right)\right) \Rightarrow V\left(t_{k}\right)<V\left(t_{k-1}\right) .
$$

where the time interval between the ROM revisions, $\delta t=t_{k}-t_{k-1}$, is chosen to satisfy $\delta t>t_{b}$.

Thus, it can be concluded that the APOD-based dynamic observer designs of (34), (38) and (49) and the APOD-based output feedback controller of (65) asymptotically stabilizes the switching closed-loop system.

By combining the APOD-based dynamic observer and APOD-based controller synthesis with $\zeta_{o}$ and $\zeta_{c}$ updating procedures, we thus obtain a hybrid output feedback control structure that guarantees that the system closed-loop stability at the desired steady state. The block diagram of the closed-loop process is presented in Fig. 2, where the connections between the proposed control structure components such as continuous point and periodic distributed measurement sensors, model reduction, dynamic observer, controller and actuators are illustrated. The proposed structure redesigns the observer/controller pair to enforce closed-loop stability whenever the ROM is revised to retain accuracy.

Remark 7. The principle of separation of estimation and control, which is relaxed in the proposed output feedback control synthesis, only holds for linear time-invariant (LTI) systems. The important consequence of the separation principle is that the controller and dynamic observer can be designed separately, i.e., the controller gain, $k$ can be computed independently of the observer gain, L. Such principle does not generally hold for linear time-variant (LTV) and nonlinear systems [16, 37]. Invoking the separation principle is a strong assumption in general nonlinear systems which limits the applicability of the resulting output feedback controller design for nonlinear systems [47]. It may lead to severe deterioration of the nominal closed-loop performance or even to closed-loop instability.

Remark 8. The controller parameters $\rho>0$ and $\chi>1$ are tuned to achieve the trade-off between desired stability region and degree of uncertainty attenuation. Using small values for these parameters enlarges the guaranteed closed-loop stability region and using large values achieves a significant degree of uncertainty attenuation in dynamic observer structure. By introducing a sufficiently small positive value of $\eta$ in the denominator of $k(\cdot)$ we circumvent the control action possible chattering behavior near the origin. In the controller structure, $c_{o}$ is also employed to tune the speed of the system convergence to the steady state.

Remark 9. Based on Assumption 3, any basis functions of slow subsystem belong in $\mathbb{P} \oplus \mathbb{Q}$. This assumption is correct only for the time intervals longer than the critical time period of $t_{b}$ needed for fast dynamics 
to be stabilized. This condition may fail due to appearance of new trends as time evolves. In such case the APOD updates the set of empirical basis functions then the ROM is revised using updated basis functions. The revised ROM is then used as the basis to redesign the controller/observer pair. These corrections will be repeated to enforce closed-loop stability during process evolution.

Remark 10. The relative significance of the empirical eigenvalues is a strong indicator that the slow subsystem is included in $\mathbb{P} \oplus \mathbb{Q}$. In general the retained snapshot ensemble size is the maximum size of the slow subsystem we can account for. We assume that at any time there are a number of eigenvalues at zero which is a requirement of all POD-based methods.

Remark 11. The presence of uncertain parameters and unmodeled dynamics, if not taken into account in the controller design, may lead to severe deterioration of the nominal closed-loop performance or even to closed-loop instability [18]. As discussed in section 3.4 the non-vanishing perturbation term in the system approximation of (64) accounts for the system parameter uncertainty beside relaxing the separation principle. The typical sources of model uncertainty which can be compensated in such non-vanishing form includes unknown or partially known time-varying process parameters, exogenous disturbances, and unmodeled dynamics [18].

\section{Application to a physico-chemical system described by the Kuramoto-Sivashinsky equation}

The effectiveness of the proposed output feedback controller in regulating physico-chemical systems described by the Kuramoto-Sivashinsky equation (KSE) is illustrated in this section. KSE has been independently derived in a wide range of physico-chemical systems, where it can adequately describe incipient instabilities. Such systems as exemplified by phase turbulence in diffusion-reaction systems, long-wave motions of falling liquid thin films, interfacial instabilities between viscous fluids and unstable flame fronts $[17,25]$. KSE with periodic boundary conditions shows a variety of dynamic behaviors, from converging to stable and unstable steady states to periodic waves and chaotic behavior, for different parameters [36]. The control problem of physico-chemical processes modeled by KSE was studied in the literature $[4,19,25,45]$.

We consider the integral controlled form of KSE as

$$
\frac{\partial \bar{x}}{\partial t}=-v \frac{\partial^{4} \bar{x}}{\partial z^{4}}-\frac{\partial^{2} \bar{x}}{\partial z^{2}}-\bar{x} \frac{\partial \bar{x}}{\partial z}+\sum_{i=1}^{l} b_{i} u_{i}(t)
$$

with periodic boundary conditions

$$
\frac{\partial^{j} \bar{x}}{\partial z^{j}}(-\pi, t)=\frac{\partial^{j} \bar{x}}{\partial z^{j}}(\pi, t), \quad j=0, \ldots, 3
$$

and initial condition

$$
\bar{x}(z, 0)=\bar{x}_{0}(z),
$$

where $\bar{x}(z, t)$ is the system variable, $u(t) \in \mathbb{R}^{l}$ denotes the vector of manipulated inputs, $t$ is the time, $z$ is the spatial coordinate, $b(z)$ describes the control actuators' distribution and $v$ is the diffusion parameter. Also the length of the spatial domain is $2 \pi$ and the domain of the process is defined on $(-\pi, \pi)$. In the above form $\mathcal{A}(z) \bar{x}=-v \frac{\partial^{4} \bar{x}}{\partial z^{4}}-\frac{\partial^{2} \bar{x}}{\partial z^{2}}$ and $\mathcal{F}(z, \bar{x})=-\bar{x} \frac{\partial \bar{x}}{\partial z}$. 
Six control actuators were considered at $L_{a}=[-\pi / 2,-\pi / 4,-\pi / 6, \pi / 5, \pi / 4, \pi / 2]$ to regulate the system dynamics. The actuators distribution function are also set as $b_{i}(z)=\delta\left(z-L_{a, i}\right)$ for $i=1, \ldots, 6$, where $\delta(\cdot)$ is the Dirac delta function. The periodic sensors distribution function is assumed as $s_{r}(z, t)=1$, i.e, the complete state profiles of the system state at ROM revisions. The important static observer requirements of available point measurements being supernumerary to the ROM dimension is circumvented using the proposed dynamic observer design (as discussed in "APOD-based dynamic observer" section). To illustrate such improvement, only one continuous point measurement sensor placed at $L_{S}=\pi / 2$ is assumed to be available. The sensor shape distribution function is chosen to be $s_{m}(z)=\delta\left(z-L_{s}\right)$ and $\bar{x}_{0}=3 \sin (z)-\cos (2 z)-\sin (5 z)+2 \cos (5 z)$ is considered as the spatially nonuniform initial condition. The open-loop spatiotemporal dynamic behavior of the system and its spatial 2-norm are presented in Fig. 3 for $v=0.4$. The control objective is to regulate the KSE at a desired spatial profile. Without loss of generality, we set the spatially uniform unstable steady state [36] of $\bar{x}_{d}(z, t)=0$ as the desired profile.

To initiate the set of empirical basis functions we consider the open-loop process $(u(t)=0)$ and collect 31 snapshots during the initial time interval of $t \in[0,2]$. By applying off-line APOD to such ensemble of snapshots we obtain 3 empirical basis functions that capture 0.99 of the ensemble energy. We consider the availability of process spatially distributed snapshots every $t_{s}=0.5$ while the point measurement from only one sensor is assumed to be available continuously. To construct the observer we applied three approaches presented in sections "pole placement", "linear optimal observer" and "linear matrix inequality". The resulting closed-loop system behavior was almost identical (when using proper sensor pole placement). For brevity only the pole placement results are presented here.

To implement the proposed APOD-based output feedback control structure on the KSE we set $\eta=$ $0.001, c_{o}=0.8, \rho=0.5, \chi=2$ and $\zeta_{i n}=1$, where $\zeta_{i n}$ indicates the initial value for $\zeta_{o}$ and $\zeta_{c}$. Fig. 4 presents the closed-loop spatiotemporal dynamic behavior of the process and its spatial 2-norm. We observe that the controller successfully stabilizes the system of (77)-(79) at $\bar{x}(z, t)=0$ and converges to zero. Fig. 5(a) shows the monitored 2-norm of error between the process modes and the estimated modes by the observer in the closed-loop time period, $t \in[2,12]$ and Fig. 5(b) presents the required control action. The monitored error is estimated based on one point sensor and it converges to zero. It is also observed that the control action converges to zero without any chattering. In Figs. 4(a), 4(b) and 5(b), $t \in[0,2]$ indicates the open-loop time period. At the end of this period, off-line APOD is used to initially construct the basis functions by collecting the snapshots. Time interval 1 in these figures shows no control action while the system is open-loop. A relatively large control action can be identified at the beginning of time interval 2 that shows the controller attempts to stabilize the process by applying large initial control action. There is another fluctuation in control actions at the beginning of time interval 3 due to $\zeta_{c}$ update. In Fig. 4(b), the temporal profile of spatial 2-norm converges to zero. We observe a peaking at the beginning of time interval 3 due to the peaking in control action. Generally, the non-smooth behavior of control action, spatial 2-norm and Lyapunov function at specific times can happen due to ROM revisions and $\zeta_{c}$ updating, where ROM revisions could include dimensionality changes. Such non-smooth behavior can be in principle compensated by incorporating input constraints considering closed-loop stability [20, 42] at the same time ensuring that the conditions set by hybrid system theory are satisfied. Fig. 6(a) and (b) present the values of the OLF and the CLF, respectively, in the closed-loop time period, $t \in[2,12]$. Note in Fig. 6(b) the increase of the CLF is due to the significant change in the behavior of the KSE. If no update was allowed the system would remain unstable. Fig. 7 shows the change in the number of basis functions during the process. The number of required empirical basis functions to capture the initial openloop dynamic of the system was three. When new patterns appeared as the process evolved the dominant 
empirical basis functions were revised to capture the process behavior. The ROM dimensionality change depends on the closed-loop process evolution and the chosen ROM design parameters, the energy fraction parameter, $\xi$. Fig. 8 shows the norm of dynamic observer gain during process evolution that is computed based on the pole placement approach to predict the modes of the system. Fig. 9 shows the profile of the updated parameters $\zeta_{c}$ and $\zeta_{o}$ based on the supervisory control such that the Lyapunov functions could satisfy the Lyapunov stability of switching systems conditions, (62). Fig. 9 shows that the CLF violated the criteria (62) at $t=3.5$ and the supervisory control updated $\zeta_{c}$ to satisfy it. Also, it indicates the OLF did not violate the hybrid system stability criteria during the closed-loop process evolution.

The temporal profile of the empirical basis functions and corresponding modes that have been estimated using the dynamic observer are presented in Figs. 10-11. It is observed that the APOD-derived basis functions are adapting with the system during the process evolution. Also, the modes of the system converge to zero; this illustrates the effectiveness of the proposed controller structure to stabilize the system at the desired steady state. The peaks in the first and second eigenmodes profiles indicate the ROM switching. Fig. 12 presents the temporal plot of the inner product of dominant empirical basis functions and their steady state spatial profile that quantifies the changes in the set of empirical basis functions during process evolution. The third basis function is not presented due to the short time-span that it was considered. The small difference for the inner product temporal profile of the first basis function shows that there were minor changes in it. The significant difference for the second temporal profile signifies the rapid changes needed in the second dominant empirical basis function during the process evolution, instigated by the nonlinear behavior of the system and the small size of the ensemble of solutions.

\section{Conclusions}

We focused on the output feedback control problem of distributed parameter systems with limited measurement sensors. A combination of a robust state feedback controller with a Luenberger-type nonlinear dynamic observer of the system states was applied to synthesize a computationally efficient control structure based on adaptive proper orthogonal decomposition. The stability of the closed-loop system was proved based on Lyapunov stability theorem for hybrid systems. The effectiveness of the proposed control method was successfully employed to stabilize a physico-chemical system described by the KuramotoSivashinsky equation when the open-loop process exhibited highly nonlinear behavior.

\section{Acknowledgment}

Financial support from the National Science Foundation, CMMI Award \# 13-00322 is gratefully acknowledged. 


\section{References}

[1] R.A. Adomaitis. A reduced-basis discretization method for chemical vapor deposition reactor simulation. Math Comput. Model, 38(1-2):159-175, 2003.

[2] A. Armaou and P.D. Christofides. Nonlinear feedback control of parabolic PDE systems with timedependent spatial domains. J. Math. Anal. \& Appl., 239:124-157, 1999.

[3] A. Armaou and P.D. Christofides. Plasma-enhanced chemical vapor deposition: Modeling and control. Chem. Eng. Sci., 54:3305-3314, 1999.

[4] A. Armaou and P.D. Christofides. Feedback control of the Kuramoto-Sivashinsky equation. Physica D, 137:49-61, 2000.

[5] A. Armaou and P.D. Christofides. Finite-dimensional control of nonlinear parabolic PDE systems with time-dependent spatial domains using empirical eigenfunctions. Int. J. Appl. Math. \& Comp. Sci., 11:287-317, 2001.

[6] A. Armaou and M.A. Demetriou. Optimal actuator/sensor placement for linear parabolic PDEs using spatial $\mathrm{H}_{2}$ norm. Chem. Eng. Sci., 61(22):7351-7367, 2006.

[7] D. Babaei Pourkargar and A. Armaou. Control of dissipative partial differential equation systems using APOD based dynamic observer designs. In Proceedings of the American Control Conference, 502-508, Washington, DC, 2013.

[8] D. Babaei Pourkargar and A. Armaou. Modification to adaptive model reduction for regulation of distributed parameter systems with fast transients. AIChE J., 59(12):4595-4611, 2013.

[9] D. Babaei Pourkargar and A. Armaou. Feedback control of linear distributed parameter systems via adaptive model reduction in the presence of device network communication constraints. In Proceedings of the American Control Conference, 1667-1673, Portland, OR, 2014.

[10] D. Babaei Pourkargar and A. Armaou. Geometric output tracking of nonlinear distributed parameter systems via adaptive model reduction. Chem. Eng. Sci., 116:418-427, 2014.

[11] D. Babaei Pourkargar and A. Armaou. Output tracking of spatiotemporal thermal dynamics in transport-reaction processes via adaptive model reduction. In Proceedings of the American Control Conference, 3364-3370, Portland, OR, 2014.

[12] D. Babaei Pourkargar and A. Armaou. APOD-based control of linear distributed parameter systems under sensor/controller communication bandwidth limitations. AIChE J., 61(2):434-447, 2015.

[13] M.J. Balas. Feedback control of linear diffusion processes. Int. J. Control, 29:523-533, 1979.

[14] M.J. Balas. Nonlinear finite-dimensional control of a class of nonlinear distributed parameter systems using residual mode filters: A proof of local exponential stability. J. Math. Anal. Appl., 162:63-70, 1991.

[15] M. Bohm, M.A. Demetriou, S. Reich, and I.G. Rosen. Model reference adaptive control of distributed parameter systems. SIAM J. Control Optim., 36 (1):33-81, 1998. 
[16] C. Brezinski. Computational aspects of linear control. Kluwer Academic Publishers, Dordrecht, 2002.

[17] H.C. Chang. Nonlinear waves on liquid film surfaces I. Flooding in vertical tube. Chem. Eng. Sci., 41:2463-2476, 1986.

[18] P.D. Christofides. Nonlinear and robust control of PDE systems. Birkhäuser, New York, 2000.

[19] P.D. Christofides and A. Armaou. Global stabilization of the Kuramoto-Sivashinsky equation via distributed output feedback control. Sys. \& Contr. Lett., 39:283-294, 2000.

[20] P.D. Christofides and N. El-Farra. Control of nonlinear and hybrid process systems: Designs for uncertainty, constraints and time-delays. Springer-Verlag, Berlin, Germany, 2005.

[21] P.D. Christofides. Robust control of parabolic PDE systems. Chem. Eng. Sci., 53(16):2949-2965, 1998.

[22] R.F. Curtain and H. Zwart. An Introduction to Infinite-dimensional Linear Systems Theory. SpringerVerlag, New York, 1995.

[23] R.A. Decarlo, M.S. Branicky, S. Pettersson, and B. Lennartson. Perspectives and results on the stability and stabilizability of hybrid systems. Proc. IEEE, 88:1069-1082, 2000.

[24] M.A. Demetriou and I.I. Hussein. Estimation of spatially distributed processes using mobile spatially distributed sensor network. SIAM J. CONTROL OPTIM., 48(1):266-291, 2009.

[25] S. Dubljevic. Boundary model predictive control of Kuramoto-Sivashinsky equation with input and state constraints. Comp. \& Chem. Eng., 34(10):1655-1661, 2010.

[26] N. El-Farra and P.D. Christofides. Robust inverse optimal control of nonlinear systems. Int. J. Rob. \& Nonl. Contr., 13:1371-1388, 2003.

[27] N. Fuji. Feedback stabilization of distributed parameter systems by a functional observer. SIAM J. Control Optim., 18:108-121, 1980.

[28] J.P. Gauthier, H. Hammouri, and S. Othman. A simple observer for nonlinear systems - Application to bioreactors. IEEE Trans. Autom. Cont., 37(6):875-880, 1992.

[29] M. Izadi and S. Dubljevic. Order-reduction of parabolic PDEs with time-varying domain using empirical eigenfunctions. AIChE J., 59(11):4142-4150, 2013.

[30] T. Kailath. Linear systems. Prentice-Hall, 1980.

[31] I. Karafyllis and C. Kravaris. Robust output feedback stabilization and nonlinear observer design. Sys. \& Contr. Lett., 54:925-938, 2005.

[32] I. Karafyllis and C. Kravaris. From continuous-time design to sampled-data design of observers. IEEE Trans. Autom. Cont., 54(9):2169-2174, 2009.

[33] I. Karafyllis and C. Kravaris. Global exponential observers for two classes of nonlinear systems. Sys. \& Contr. Lett., 61(7):797-806, 2012. 
[34] N. Kazantzis and C. Kravaris. Nonlinear observer design using Lyapunov's auxiliary theorem. Sys. \& Contr. Lett., 34:241-247, 1998.

[35] H. Keller. Nonlinear observer design by transformation into a generalized observer canonical form. Int. J. Contr., 46(6):1915-1930, 1987.

[36] I.G. Kevrekidis, B. Nicolaenko, and J.C. Scovel. Back in the saddle again: A computer assisted study of the Kuramoto-Sivashinsky equation. SIAM J. Appl. Math., 50:760-790, 1990.

[37] H.K. Khalil. Nonlinear systems. Prentice-Hall, New Jersey, 2002.

[38] M. Krstic and A. Smyshlyaev. Adaptive boundary control for unstable parabolic PDEs - Part I: Lyapunov design. IEEE Trans. Autom. Cont., 53:1575-1591, 2008.

[39] Y.H. Lin and R.A. Adomaitis. Simulation and model reduction methods for an RF plasma glow discharge. J. Ccmput. Phys., 171(2):731-752, 2001.

[40] P. Lu, J.H. Edgar, O.J. Glembocki, P.B. Klein, E.R. Glaser, J. Perrin, and J. Chaudhuri. High-speed homoepitaxy of $\mathrm{SiC}$ from methyltrichlorosilane by chemical vapor deposition. J. Cryst. Growth, 285(4):506-513, 2005.

[41] D.G. Luenberger. Observing the state of a linear system. IEEE Trans. Milit. Electr., 8:74-80, 1963.

[42] P. Mhaskar and A. Kennedy. Robust model predictive control of nonlinear process systems: Handling rate constraints. Chem. Eng. Sci., 63:366-375, 2008.

[43] H. Michalska and D.Q. Mayne. Moving horizon observers and observer-based control. IEEE Trans. Autom. Cont., 40(6):995-1006, 1995.

[44] J. Ng and S. Dubljevic. Optimal boundary control of a diffusion-convection-reaction PDE model with time-dependent spatial domain: Czochralski crystal growth process. Chem. Eng. Sci., 67(1):111-119, 2012.

[45] S. Pitchaiah and A. Armaou. Output feedback control of distributed parameter systems using adaptive proper orthogonal decomposition. Ind. Eng. Chem. Res., 49:10496-10509, 2010.

[46] S. Pitchaiah and A. Armaou. Output feedback control of dissipative PDE systems with partial sensor information based on adaptive model reduction. AICHE J., 59(3):747-760, 2013.

[47] C. Qian and W. Lin. Output feedback control of a class of nonlinear systems: A nonseparation principle paradigm. IEEE Trans. Autom. Cont., 47(10):1710-1715, 2002.

[48] D.L. Russell. Controllability and stabilizability theory for linear partial differential equations: Recent progress and open questions. SIAM Review, 20:639-739, 1978.

[49] C. Scherer, P. Gahinet, and M. Chilali. Multiobjective output-feedback control via LMI optimization. IEEE Trans. Autom. Cont., 42:896-911, 1997.

[50] L. Sirovich. Turbulence and the dynamics of coherent structures: Parts I, II and III. Quarterly of Applied Mathematics, XLV:561-590, 1987. 
[51] A. Smyshlyaev and M. Krstic. Backstepping observers for a class of parabolic PDEs. Sys. \& Contr. Lett., 54(7):613-625, 2005.

[52] A. Smyshlyaev and M. Krstic. Adaptive boundary control for unstable parabolic PDEs - Part II: Estimation-based designs. Automatica, 43 (9):1543-1556, 2007.

[53] A. Smyshlyaev and M. Krstic. Adaptive control of parabolic PDEs. Princeton Univ. Press, Princeton, NJ, 2010.

[54] E. Sontag. A universal construction of Artsteins theorem on nonlinear stabilization. Sys. \& Contr. Lett., 13:117-123, 1989.

[55] M. Soroush. Nonlinear state-observer design with application to reactors. Chem. Eng. Sci., 52:387404, 1997.

[56] R. Temam. Infinite-dimensional dynamical systems in mechanics and physics. Springer-Verlag, New York, 1988.

[57] F.E. Thau. Observing state of nonlinear dynamic-systems. Int. J. Contr., 17(3):471-479, 1995.

[58] A. Theodoropoulou, R.A. Adomaitis, and E. Zafiriou. Model reduction for optimization of rapid thermal chemical vapor deposition systems. IEEE Trans. Semicond. Manuf., 11(1):85-98, 1998.

[59] D. Ucinski. Measurement Optimization for Parameter Estimation in Distributed Systems. Technical University Press, Zielona Gora, 1999.

[60] D. Ucinski. Optimal Measurement Methods for Distributed Parameter System Identification. CRC Press, Boca Raton, 2005.

[61] A. Varshney, S. Pitchaiah, and A. Armaou. Feedback control of dissipative distributed parameter systems using adaptive model reduction. AICHE J., 55:906-918, 2009.

[62] R.J. Veillette. Reliable linear-quadratic state feedback control. Automatica, 31:137-143, 1995.

[63] C.Z. Xu, P. Ligarius, and J.P. Gauthier. An observer for infinite-dimensional dissipative bilinear systems. Computers Math. Applic., 29(7):13-21, 1995. 




Figure 1: Flow chart of adaptive model reduction methodology. Blue boxes denote algorithm I/Os and green decision steps. 


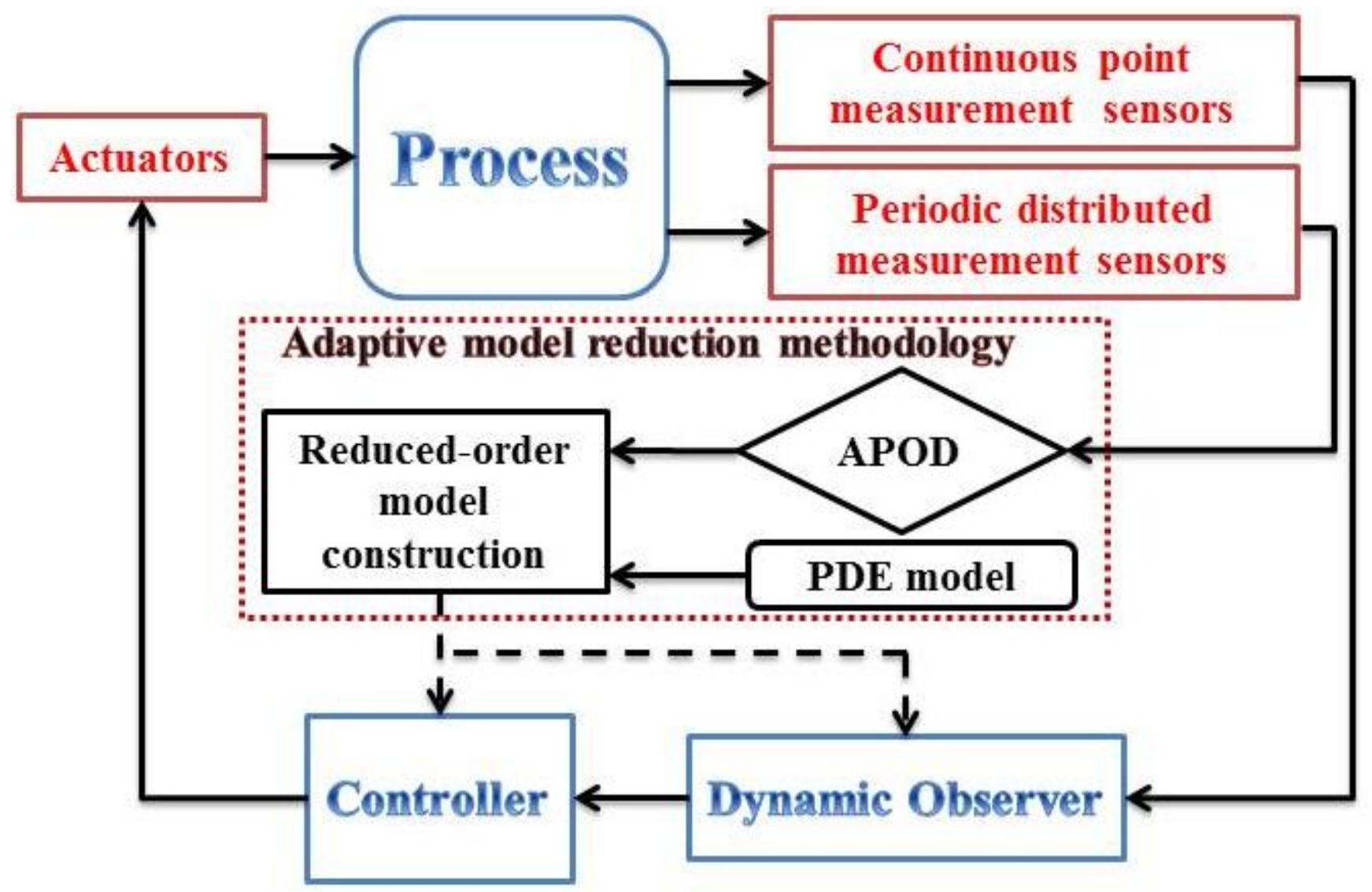

Figure 2: Process operation block diagram under the proposed controller structure. 

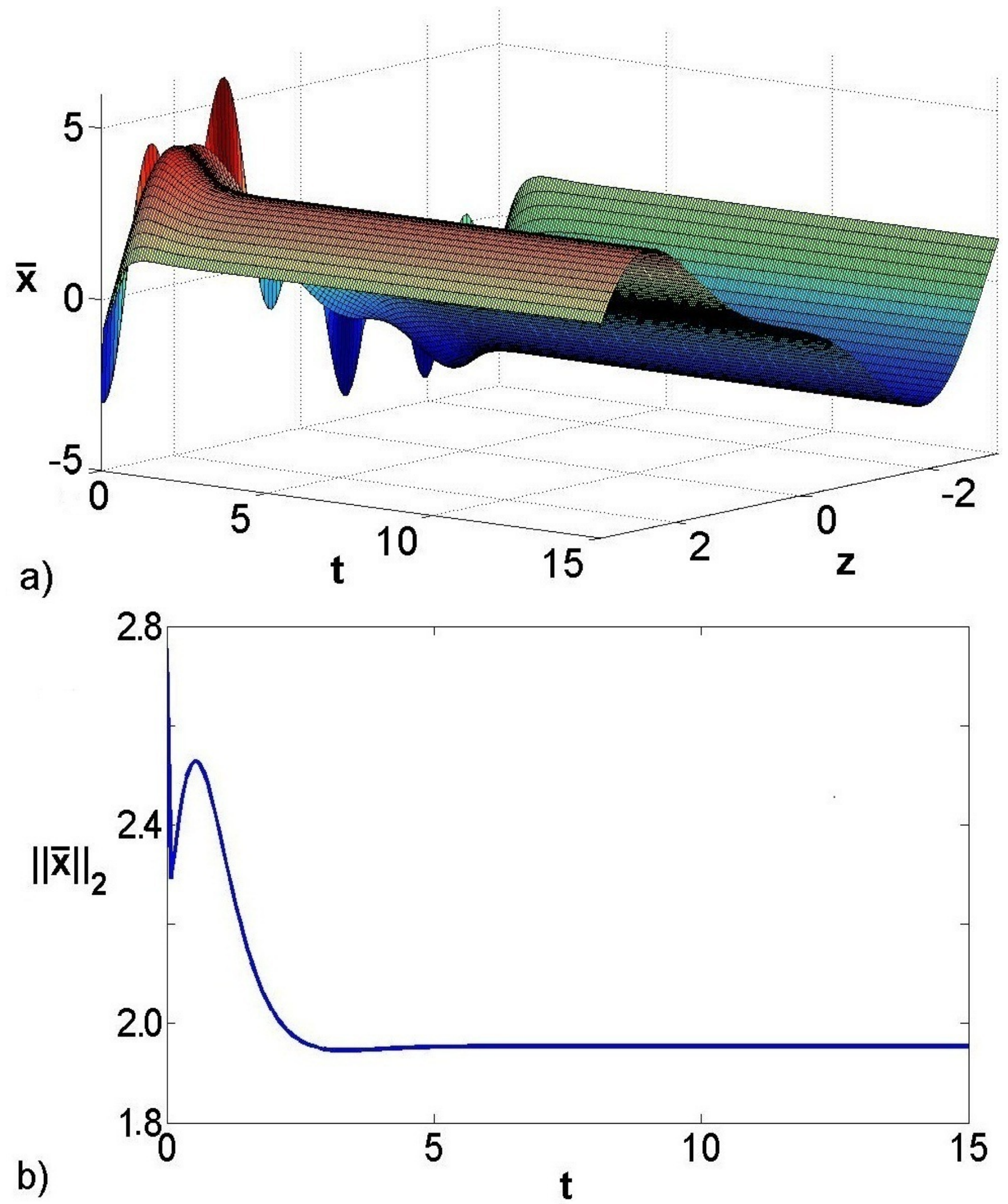

Figure 3: Open-loop spatiotemporal profile of (a) state of the system and (b) 2-norm of the state for $v=0.4$. 

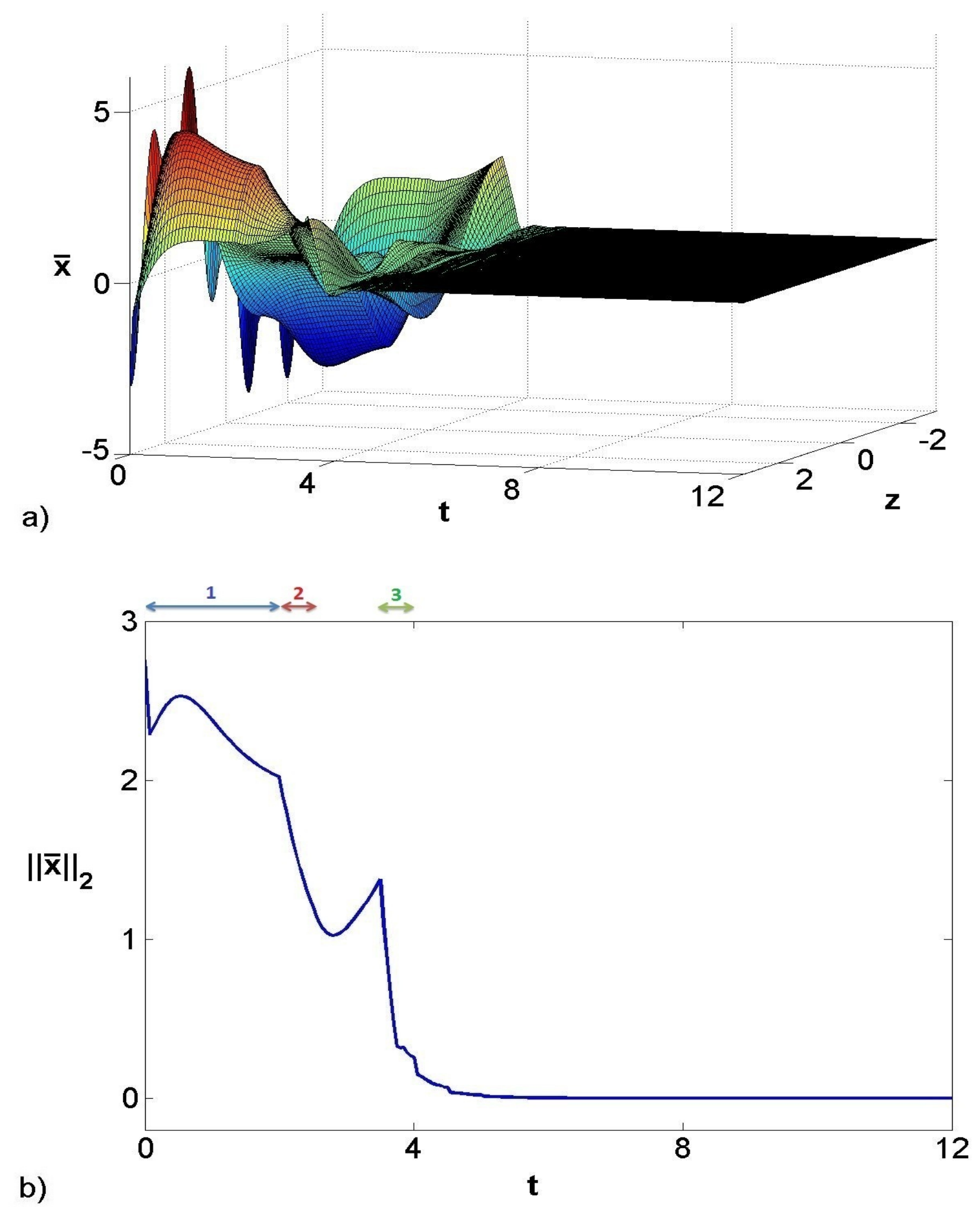

Figure 4: Closed-loop system temporal profile of (a) the state spatial profile, (b) 2-norm of the state. 

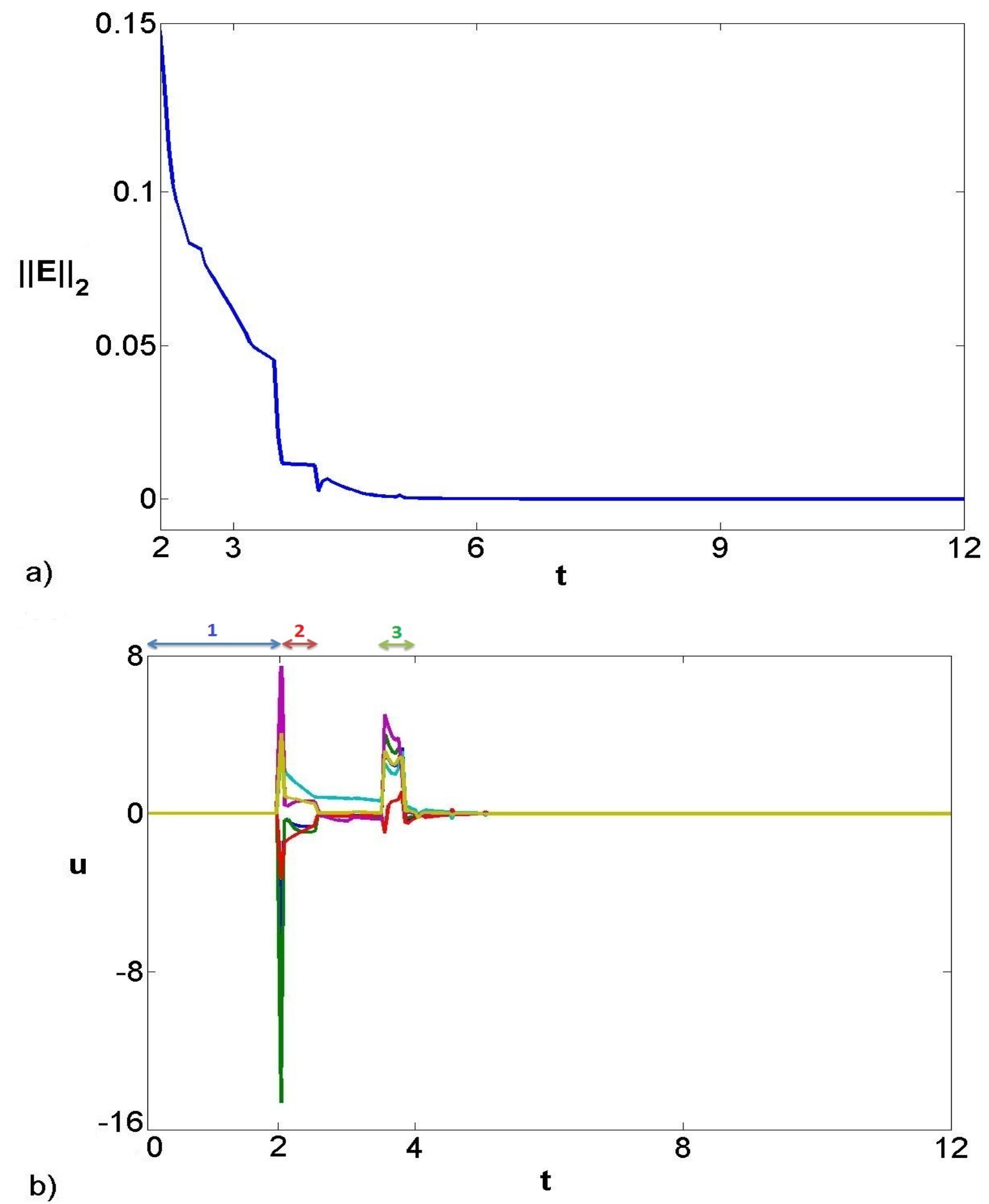

Figure 5: Closed-loop system temporal profile of (a) monitored norm of error between the real system and the observer from point measurement sensor, and (b) control action. 

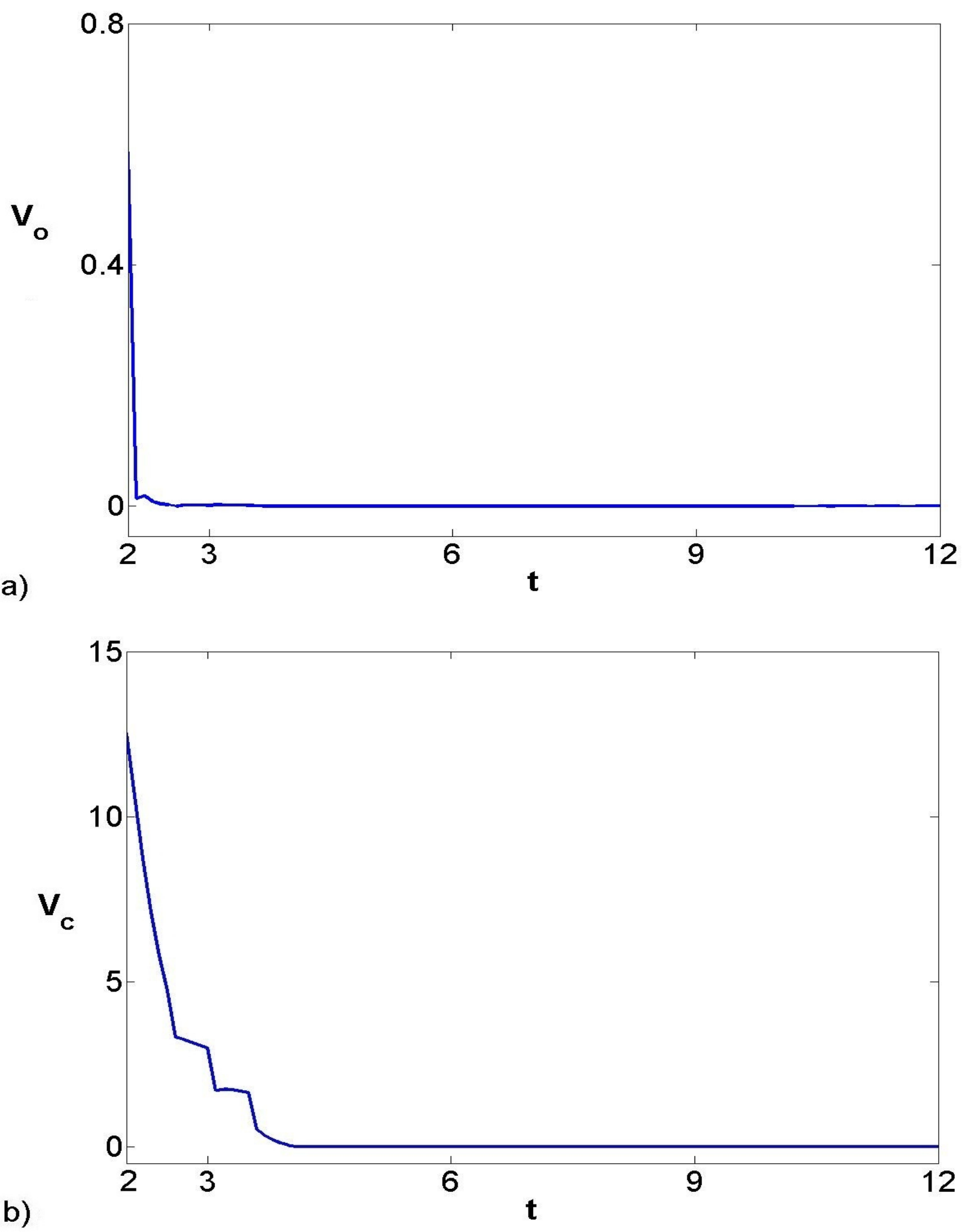

Figure 6: Closed-loop system temporal profile of (a) OLF, and (b) CLF. 


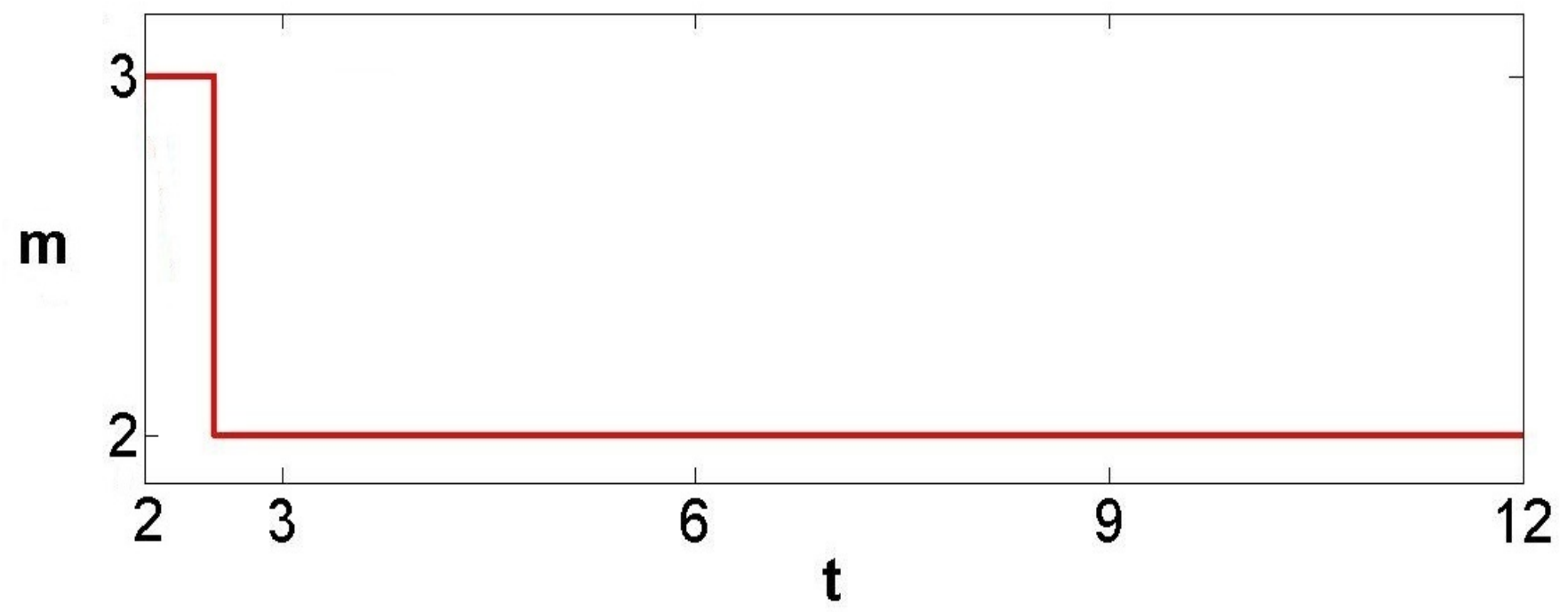

Figure 7: Number of basis functions. 




Figure 8: The 2-norm of the observer gains. 


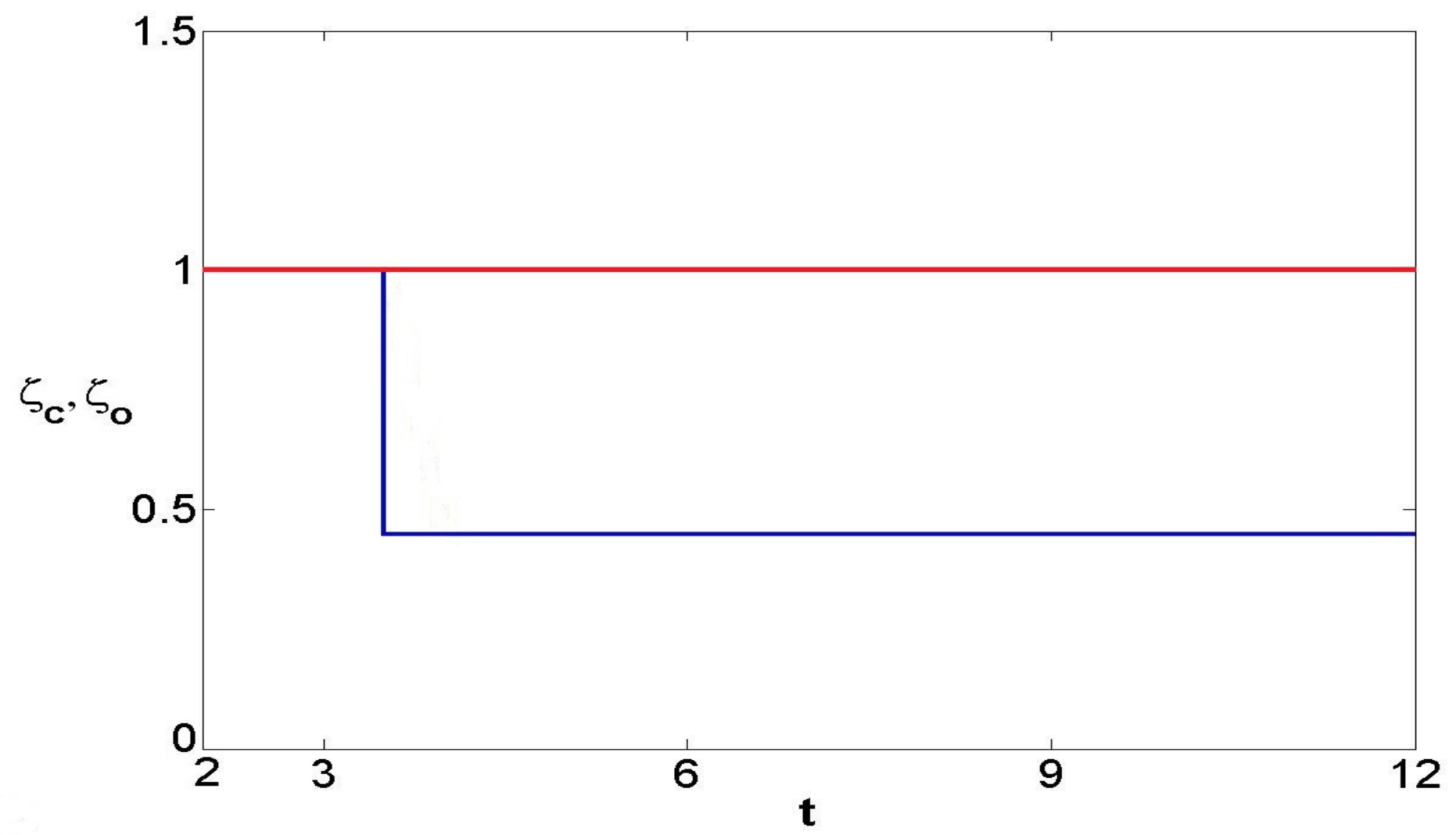

Figure 9: Temporal profile of updated parameters $\zeta_{c}$ (blue line) and $\zeta_{o}$ (red line) based on supervisory control. 


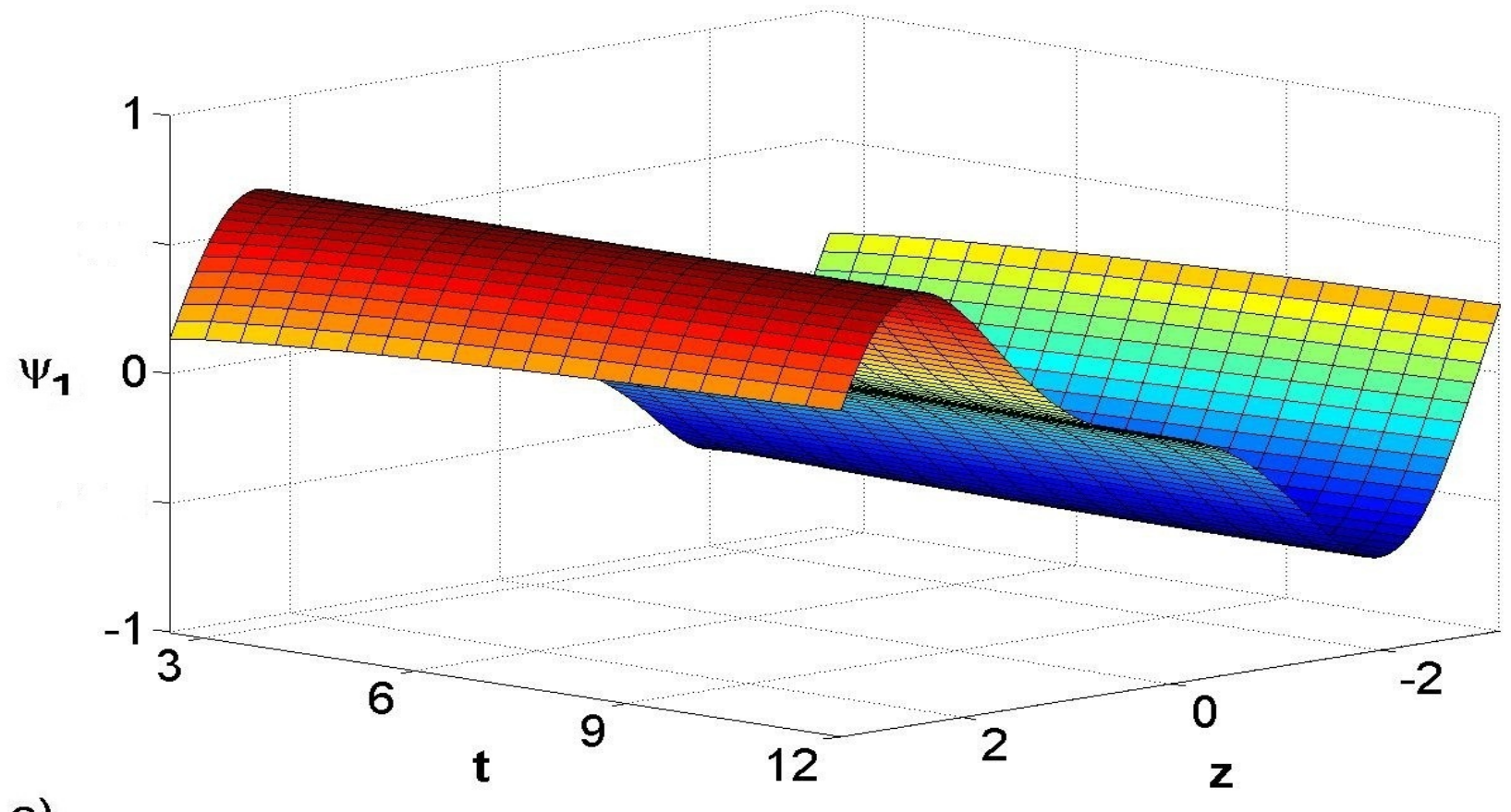

a)

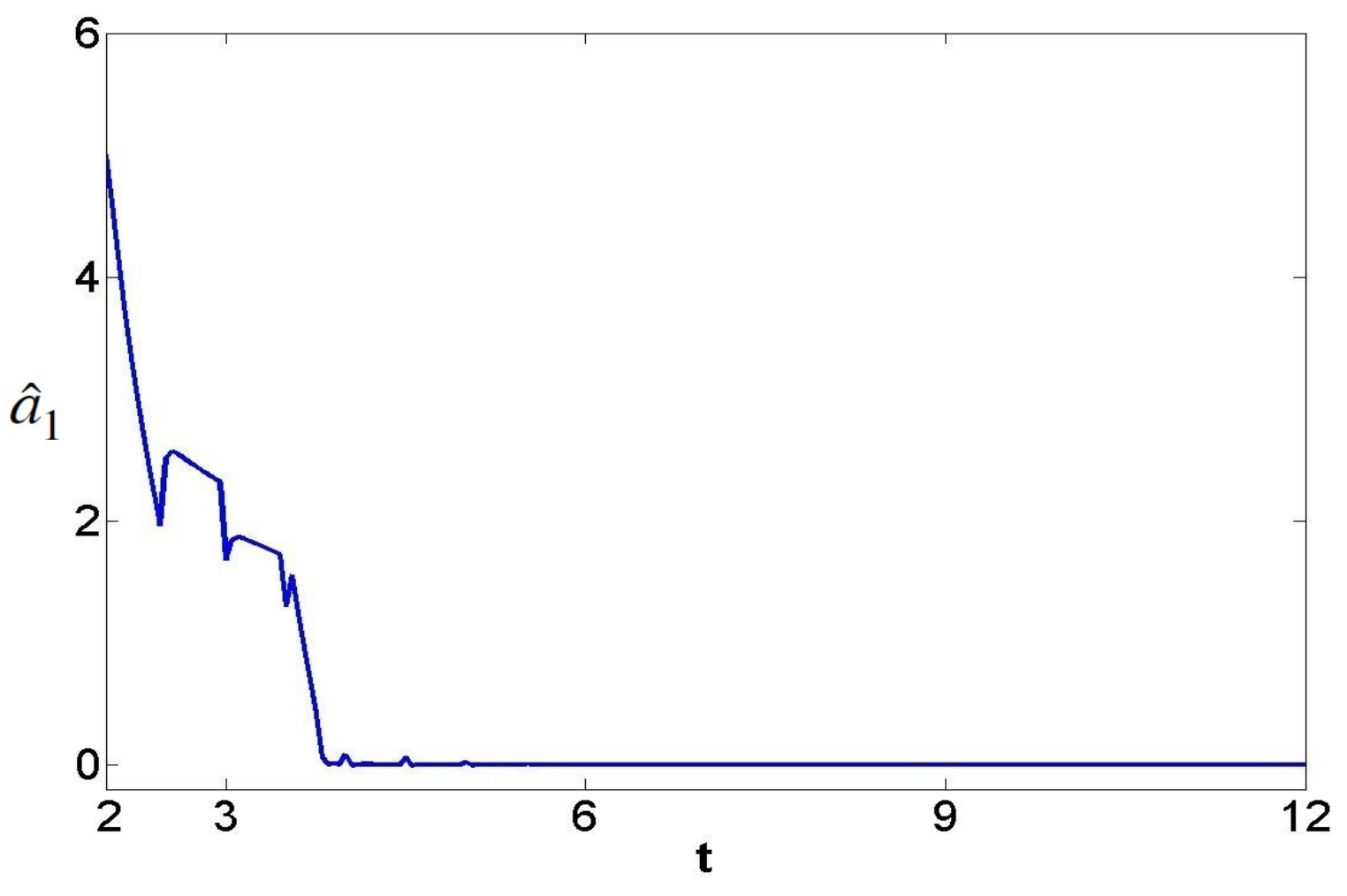

Figure 10: Temporal profile of (a) the first basis function and (b) First eigenmode. 

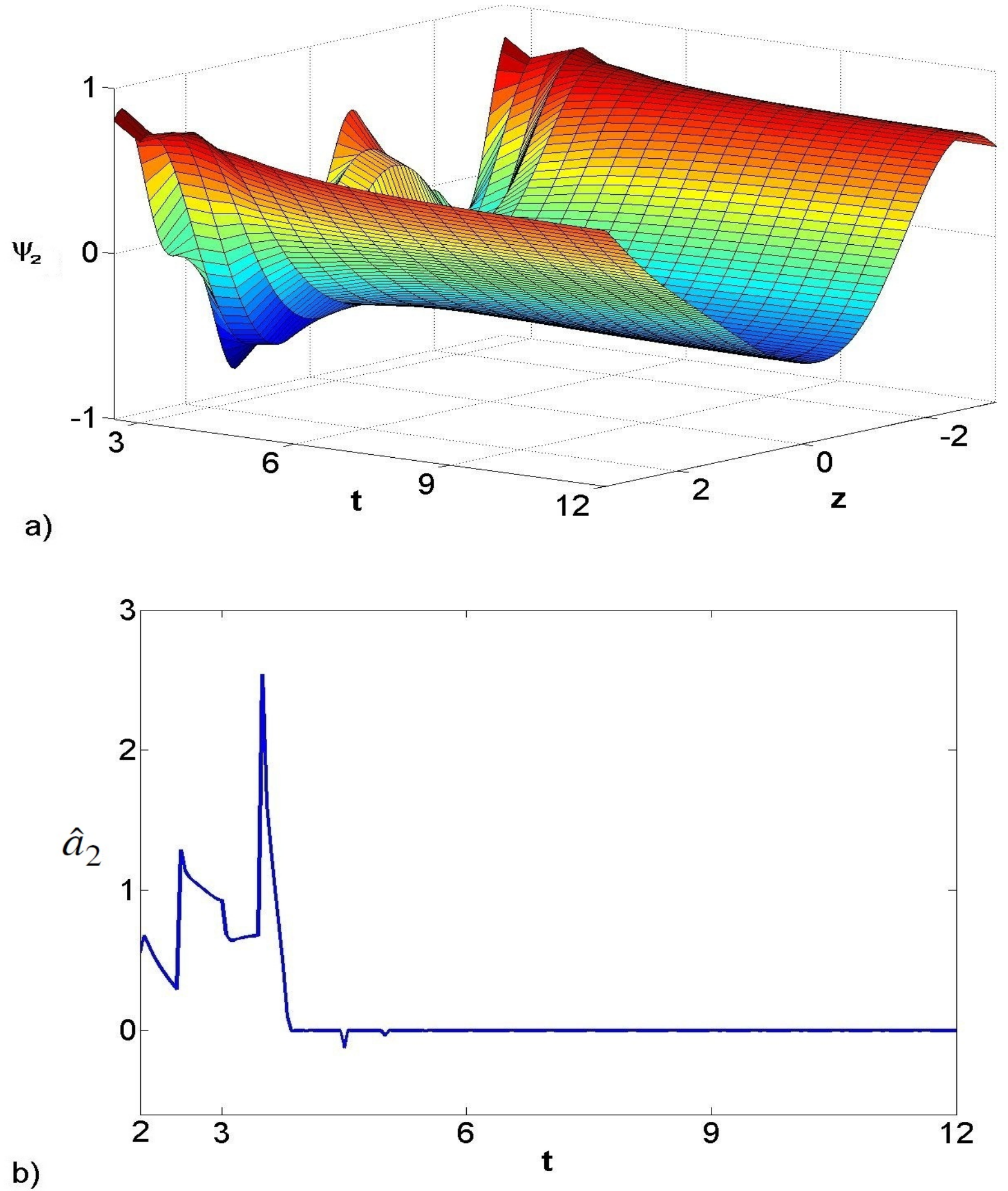

Figure 11: Temporal profile of (a) the second basis function and (b) Second eigenmode. 


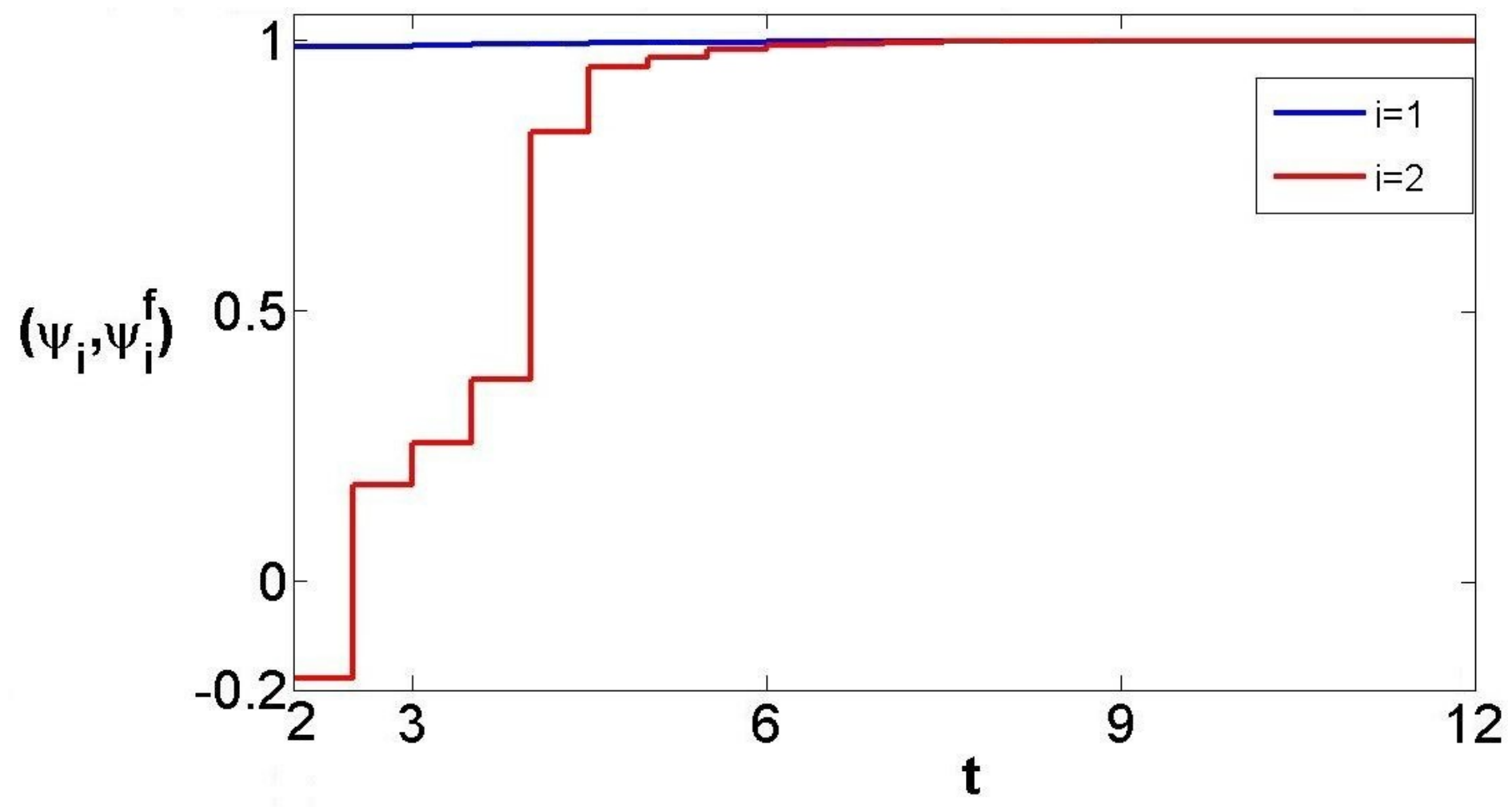

Figure 12: Temporal profile of the inner product of dominant empirical basis functions with respect to their final time spatial profile. 